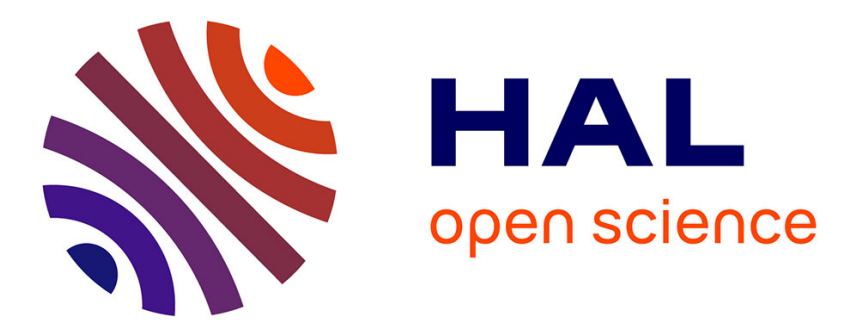

\title{
Interrogations théoriques autour de la propriété et du foncier en Europe
}

Gérard Chouquer

\section{To cite this version:}

Gérard Chouquer. Interrogations théoriques autour de la propriété et du foncier en Europe. Gérard Chouquer; Marie-Claude Maurel. Les mutations récentes du foncier et des agricultures en Europe, Presses universitaires de Franche-Comté, pp.229-256, 2019, Les Cahiers de la MSHE, 978-2-84867-641-

8. hal-03248771

\section{HAL Id: hal-03248771 \\ https://univ-fcomte.hal.science/hal-03248771}

Submitted on 3 Jun 2021

HAL is a multi-disciplinary open access archive for the deposit and dissemination of scientific research documents, whether they are published or not. The documents may come from teaching and research institutions in France or abroad, or from public or private research centers.
L'archive ouverte pluridisciplinaire HAL, est destinée au dépôt et à la diffusion de documents scientifiques de niveau recherche, publiés ou non, émanant des établissements d'enseignement et de recherche français ou étrangers, des laboratoires publics ou privés. 


\section{Les mutations récentes}

\section{du foncier et des agricultures en Europe}

sous la direction de

Gérard CHOUQUER

et Marie-Claude MAUREL

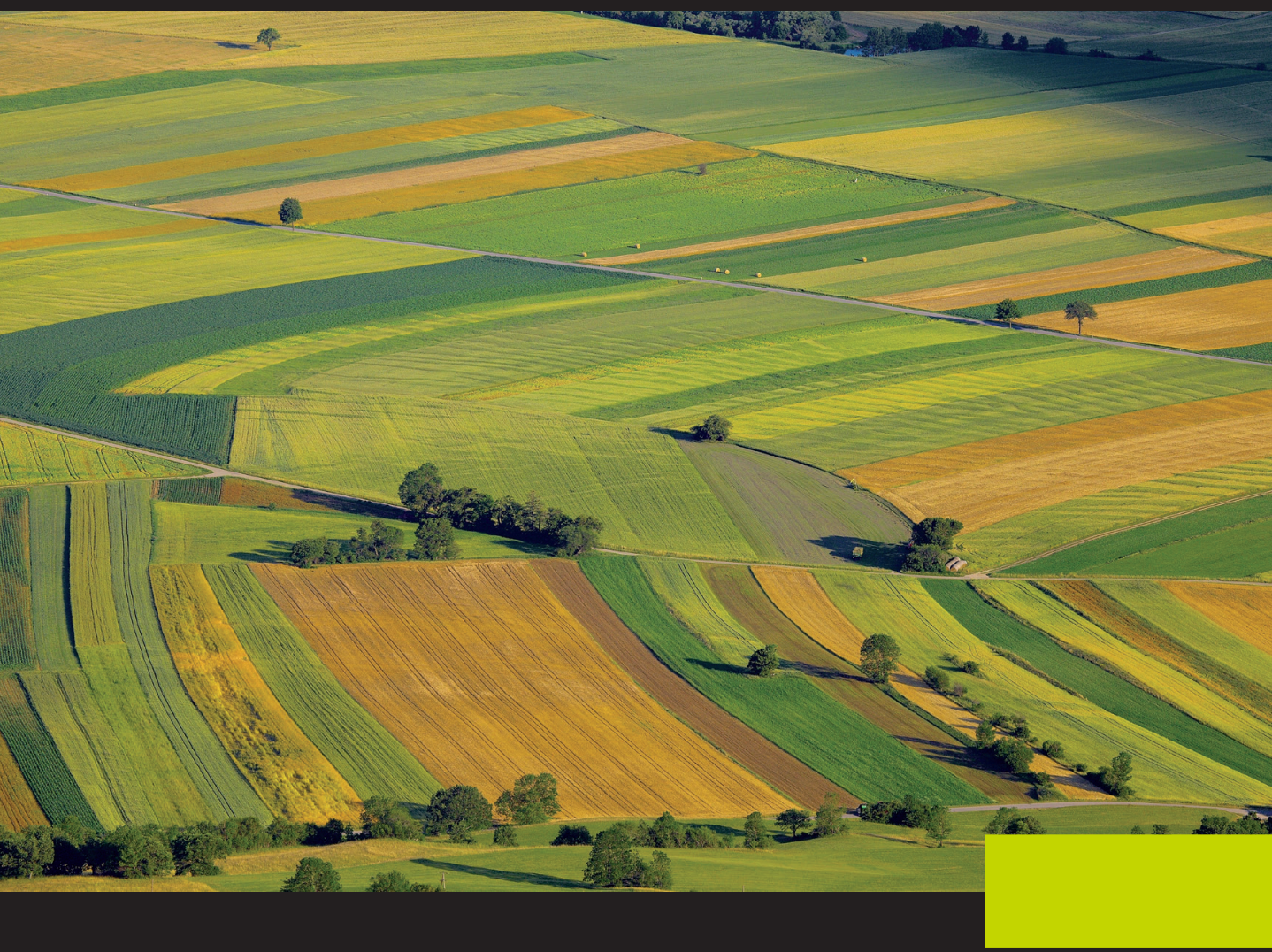

Presses universitaires de Franche-Comté

Presses universitaires Sun Yat-sen de Guangzhou 
Illustration de couverture :

Vue aérienne des champs agricoles

Source : (C) 123RF/Peter Gudella 


\section{LES MUTATIONS RÉCENTES DU FONCIER ET DES AGRICULTURES EN EUROPE}




\section{PRESSES UNIVERSITAIRES DE FRANCHE-COMTÉ}

\section{$\mathrm{N}^{\circ} 1445$}

\section{Collection « LES CAHIERS DE LA MSHE LEDOUX » dirigée par Philippe Barral \\ $n^{\circ} 35$}

Série "Normes et pratiques foncières et agricoles dans le monde » éditée en version originale chinoise par les Presses universitaires Sun Yat-sen de Canton, puis en version française par les Presses universitaires de Franche-Comté Responsable : Gérard Chouquer

$$
\mathrm{n}^{\circ} 1
$$

\section{MSHE Claude Nicolas Ledoux, USR 3124 \\ 30-32, rue Mégevand \\ 25030 Besançon cedex}

\footnotetext{
(C) PRESSES UNIVERSITAIRES DE FRANCHE-COMTÉ

ET PRESSES UNIVERSITAIRES SUN YAT-SEN DE CANTON - 2018

Mise en page et suivi éditorial de la version française : Marie Gillet
}

ISBN : 978-2-84867-641-8 - EAN : 9782848676418

ISSN : 1771-8988 


\title{
LES MUTATIONS RÉCENTES DU FONCIER ET DES AGRICULTURES EN EUROPE
}

\author{
sous la direction de \\ Gérard Chouquer et Marie-Claude Maurel
}

\begin{abstract}
auteurs :
Guihem Anzalone, Gilles Bazin, Hubert Bosse-Platière,

Pascal Chevalier, Gérard Chouquer, Hubert Cochet, Sophie Devienne, Clémence Guimont, Bertrand Hervieu, Guillaume Lacquement, Marie-Claude Maured, Geneviève NGuyen, François Purseigle, Aurélie Trouvé, Bruno Villalba
\end{abstract}

Ouvrage publié avec le concours des Pressses universitaires Sun Yat-sen de Canton (Chine) 
La création de cette collection est une opportunité due à la volonté des Presses de l'Université Sun Yat-sen de Guangzhou (province de Guangdong). Grâce à la présence d'un important département de français dans cette université, les Presses universitaires Sun Yat-sen ont choisi de s'investir dans la mise à disposition du public chinois de traductions d'ouvrages originaux de langue française sur les questions foncières, agricoles et environnementales, qui permettent à la recherche et à l'enseignement universitaire chinois de disposer d'ouvrages équilibrant les traductions d'ouvrages anglo-saxons dont ils disposent déjà.

Lors de la mise en route, avant le lancement de cette collection, de la traduction de deux ouvrages déjà publiés (Bertrand Hervieu et François Purseigle, 2013, Sociologie des mondes agricoles, Paris, Armand Colin; Gérard Chouquer, 2012, Terres porteuses, Arles, Actes Sud), il a été décidé d'un commun accord de faire porter l'effort sur la publication d'ouvrages inédits dont la version originale sera publiée dans une collection nouvelle en Chine puis, quasi simultanément, en version française dans une série également nouvelle de la collection des "Cahiers de la Maison des sciences de l'homme et de l'environnement » de Besançon, en raison de la cession de droits consentie par les Presses universitaires Sun Yat-sen aux Presses universitaires de Franche-Comté.

L'association France internationale pour l'expertise foncière, FIEF (Ordre des géomètres-experts) a manifesté son intérêt pour cette initiative et en a facilité la réalisation, notamment en apportant un concours juridique à la rédaction des contrats et en proposant son réseau d'experts fonciers.

Les Presses universitaires Sun Yat-sen de Guangzhou et les Presses universitaires de Franche-Comté, ont confié la direction de la collection ou de la série à Gérard Chouquer (ancien directeur de recherches au CNRS; expert foncier et secrétaire de FIEF), et ont sollicité Marie-Claude Maurel (ancienne directrice du département Sciences de l'homme et de la société du CNRS, directrice d'études à l'EHESS) pour qu'elle assure la suppléance de cette direction et apporte son conseil à la conduite du projet. 


\section{Remerciements}

Les Presses universitaires Sun Yat-sen de Guangzhou et les Presses universitaires de Franche-Comté remercient France internationale pour l'expertise foncière (Ordre des géomètres-experts, 40 avenue Hoche, 75008 Paris, France), pour l'aide proposée lors la réalisation de cet ouvrage »

Gérard Chouquer et Marie-Claude Maurel remercient Monsieur Xu Jing, directeur des Presses universitaires Sun Yat-sen de Guangzhou et Madame Zeng Xiaoyang, directrice du département de français de cette université, pour l'accueil qu'ils ont réservé à ce projet et pour l'extrême qualité des échanges lors du suivi de cette réalisation.

Ils remercient également Jean-Paul Barrière, directeur des Presses universitaires de Franche-Comté, et Philippe Barral, directeur de la Maison des sciences de l'homme et de l'environnement Claude Nicolas Ledoux (Besançon), pour leur accueil et leurs encouragements. 


\title{
Chapitre 10
}

\section{Interrogations théoriques autour de la propriété et du foncier en Europe}

\author{
Gérard ChOUQuer
}

L'objet de ce chapitre est de présenter au lecteur non spécialiste un état de la réflexion sur la notion de propriété, le modèle qu'elle représente et les effets que cette réflexion peut avoir sur la mise en pratique du droit. Pour situer simplement les choses, entre ceux qui prennent acte de «l'empire de la propriété $»^{1}$ et de son extension, et ceux qui font du "propriétarisme » l'idéologie à combattre, toute une gamme de positions classiques ou alternatives se développe, proposant divers argumentaires.

Après avoir caractérisé les systèmes fonciers hérités de l'histoire européenne (1) et leurs différences de conception (2), cette contribution se propose de décrire l'état du débat autour de la notion de propriété foncière, à travers la notion de crise de l'idée de propriété (3), des approches libérales ou alternatives proposées (4), enfin, de mettre en évidence les changements que de nouvelles pratiques apportent aux théories (5).

\section{Caractérisation des régimes fonciers hérités de l'Europe}

Pour qualifier un régime foncier*, trois questions importent : quel est le statut de la propriété ? Est-elle formalisée ? Et quel est son rapport éventuel avec le Cadastre?

\section{Le foncier* dans le droit dit de common law}

Les pays de common law, c'est-à-dire en Europe uniquement les îles Britanniques, sont ceux qui conservent l'apparence de la structure coutumière et féodale d'Ancien Régime*, forme héritée de leur histoire, et qui, pour la définition de leurs normes, privilégient la jurisprudence et non le Code (Papandréou-Deterville, 2004). Si l’on parle

1. Voir le volume publié par Éric de Mari et Dominique Taurrisson-Mouret en novembre 2016 sur ce thème. 
d'apparence, c'est parce qu'il ne faut pas durcir inutilement la forme du système : par exemple, si le droit anglais conserve la superposition de l'éminent* et de l'utile*, si l'on emploie toujours les mots de fief* et de tenure*, comme dans l'Ancien Régime, les garanties modernes offertes au propriétaire utile sont telles que son droit n'est pas un droit mineur et qu'il n'est plus un tenancier médiéval!

Le récit* historique idéologique de l'Angleterre repose sur le fil directeur suivant: le droit dit common law, qui est fondamentalement un droit issu de la coutume, aurait connu une « continuité organique » (Berman, 2011), c'est-à-dire un développement harmonieux et endogène, parce qu'il aurait successivement résisté aux droits " étrangers » qu'étaient le droit canonique catholique du Moyen Âge et le droit romain. Dans ce récit, les épisodes monarchiques des Tudors puis des Stuarts sont lus comme étant des moments despotiques ayant interrompu le cours de cette continuité organique.

En mettant en avant l'idée que le droit d'une nation est principalement le produit de son histoire, cela permettait de lutter contre l'equity, ce corps de règles et d'instruments dont la monarchie avait usé pour affaiblir les cours de common law, et renforcer son pouvoir. Elle permettait d'affirmer le caractère sui generis et articulé du droit foncier* et de l'aristocratie à travers la structure seigneuriale du manoir*. Voilà pourquoi le droit anglais common law, le plus auto-organisationnel des droits ou celui qui se prétend tel, offre une identité originale et s'oppose, par principe, à tout processus d'institution, c'est-à-dire de définition préalable ou "par le haut », ce qui est le cas lorsqu’on édicte un code. Le droit common law croît mais ne naît pas : il ne s'assigne pas d'origine ; et s'il se développe et même change, c'est dans le respect de son identité. Il est ainsi plus un droit jurisprudentiel qu'un droit de législateur.

On tient sans doute là une explication de la séduction qu'exerce le droit anglais de par le monde, en lien avec le thème des communs et la défense des communautés, puisqu'il ne rompt pas avec la coutume médiévale, et ne pose pas le processus de « modernisation » comme étant un passage obligé, ce qui est le cas du droit latin réinterprété depuis l'expérience révolutionnaire française.

Cependant, l'histoire des évolutions du droit foncier anglo-saxon est intéressante car elle est contradictoire et ne s'inscrit pas uniquement dans cette représentation. Car, à côté de la résistance opiniâtre de l'aristocratie à la pression modernisante exercée sur elle, et, par conséquent, aux succès limités remportés par l'État anglais dans ce domaine, on constate aussi de très récentes évolutions qui font entrer le Royaume-Uni dans une nouvelle époque de son histoire foncière : tel est le cas de l'évolution de ce pays vers le cadastre (Kain et al., 2008), ce qui est un changement notable dans un pays qui a toujours fait de la résistance au cadastre et à l'enregistrement de la propriété foncière une base idéologique et une règle de conduite.

\section{Les pays de droit foncier* formaliste et codifié}

Les pays qui adoptent la base civiliste* latine sont ceux qui se situent dans une double attitude, vis-à-vis de Rome et vis-à-vis des anciens régimes. Ces pays se réclament, plus que d'autres, du droit romain, mais à travers des réinterprétations de ce droit qui ont eu lieu à l'époque de Justinien (Maganzani, 2007), à partir du XI siècle, puis encore aux $\mathrm{XVI}^{\mathrm{e}}-\mathrm{XVIII}{ }^{\mathrm{e}}$ siècles lorsqu'il s'agissait d'essentialiser le propriétaire à travers la promotion de trois termes qui décrivent la plénitude de ses droits : usus (droit d'user), fructus (droit de retirer les fruits), abusus* (droit d'agir sans limites). 
Ensuite, ces pays posent le principe d'une rupture avec l'Ancien Régime*, idéal qui a été porté au paroxysme par l'idéologie de la Révolution française. Le régime de la propriété est conditionné par la définition de la citoyenneté et le fait qu'à partir de la Révolution française, plus aucun lien de dépendance ne doit subsister entre les personnes. La propriété ne peut donc plus être divisée entre éminent et utile*, c'est-à-dire entre un seigneur et un tenancier, ce qui explique la résistance des auteurs de langue française à user, aujourd'hui, du terme de " tenure* », plus courant en langue anglaise. D'autre part, la propriété est définie de façon consensuelle, expression de la volonté des parties, sans formalisme, ne nécessitant, pour faire preuve, ni d'un passage devant le juge (comme c'était le cas dans la procédure de traditio ou transfert de la propriété du citoyen romain), ni d'un enregistrement dans un livre foncier (comme c'est le cas dans le système germanique).

Aujourd'hui, l'une des questions conceptuelles qu'affrontent les chercheurs est celle de savoir comment diviser à nouveau la propriété sans retomber dans la distinction entre éminent et utile et dans les liens de dépendance ? La réponse n'est pas encore clairement apportée et c'est peut-être une des raisons pour lesquelles les solutions alternatives se multiplient mais ne s'imposent pas.

La sécurité est apportée par un système dit de «publicité foncière* », progressivement défini aux $\mathrm{XIX}^{\mathrm{e}}$ et $\mathrm{XX}^{\mathrm{e}}$ siècles et fixé, en France, en 1955. Il s'agit d'une publicité qui ne fait que constater la propriété. En effet, en France et dans nombre de pays qui ont adopté le Code civil de 1804, c'est l'accord des parties, exprimé devant le notaire, qui fonde la transmission. C'est du jour de ce constat, donc avant même toute publicité foncière*, que le changement de propriétaire produit ses effets juridiques. Enfin, le lien avec le Cadastre est assez ténu : en France, le cadastre est uniquement fiscal et n'est pas constitutif de la propriété : c'est-à-dire que le cadastre est un outil du ministère des Finances.

La logique française ne manque pas de contradictions. D’un côté elle est profondément consensualiste, subsidiaire (peut-on imaginer plus « local» et plus « consensuel» que l'étude du notaire au sein de laquelle l'accord des parties suffit à transférer la propriété ?) et désormais décentralisée. Mais d'un autre, elle hérite et transmet, à travers le poids de l'Administration, le souci de ne pas perdre les instruments d'un contrôle approfondi, quelquefois très bureaucratique. S'il y a, à côté de la souplesse, une rigidité à la française, c'est bien dans cette hésitation permanente entre, d'un côté, l'observation des réalités et leur prise en compte progressive dans une espèce d'attitude libérale ne se donnant pas de limites préalables, et, d'un autre côté, la volonté toujours présente - qu'on la nomme colbertiste, jacobine ou centralisatrice ${ }^{2}-$ d'en avoir une vision d'ensemble préalable qui justifierait la mise en œuvre de toutes les rationalités. En raison de cet héritage, la France, sans doute plus que d'autres pays, souffre du fait de n'avoir qu'imparfaitement réussi une espèce d'abornement général de ses activités sociales, si on me permet cette métaphore d'arpentage ${ }^{3}$, et de devoir aujourd'hui concéder le fait que certaines évolutions la rattrapent. Il lui en reste une prudence vis-à-vis de tout ce qui peut déréguler, la

2. Ces trois adjectifs se rapportent : 1) au ministre de Louis XIV, Colbert, grand artisan de la construction de l'autorité monarchique centrale au $\mathrm{XVII}^{\mathrm{e}}$ siècle ; 2) aux jacobins, fraction politique qui, pendant la Révolution française de 1789, défendait la centralisation et s'opposaient aux fédéralistes girondins ; 3) à la tradition de l'Administration française de faire remonter au niveau central la moindre décision locale.

3. L’abornement ou bornage général était la grande question des campagnes au Xix ${ }^{e}$ siècle en France, et il ne fut jamais réalisé. 
prudence de l'Administration n'étant pas moindre que la réticence du citoyen, la cautèle du juriste ou encore le refus du syndicaliste. L'exemple de l'histoire de l'adoption, timide et discutée, de la fiducie* en droit français illustre cette forte hésitation.

D'autre part, l'adoption de cette forme citoyenne et consensuelle de propriété n'a pas fait disparaître les inégalités sociales puisque si chacun peut, en droit, devenir propriétaire, tout le monde ne le devient pas, en fait. Ainsi s'explique cette tendance, lourde par les effets qu'elle ne cesse de produire, qui habite les esprits, celle d'un inachèvement (social) de la Révolution française, source d'un courant politique polymorphe qui ne cesse de chercher comment la parachever pour certains, ou même la subvertir, pour d'autres.

Avec des nuances qu'il serait intéressant de relever et de commenter en détail, le régime foncier* latin est répandu dans les pays de l'Europe méridionale : Espagne, Italie, Portugal, Bulgarie, Roumanie (en partie), Grèce, et présent aussi dans le Benelux. La principale nuance étant que dans certains de ces pays (Espagne, Bulgarie ou Grèce) le registre foncier dépend du ministère de la Justice et apparente donc ces systèmes au cas germanique.

Viennent ensuite les pays, dits de tradition germanique, qui adoptent une base civiliste* mais qui le font dans une conception articulant le consensualisme (la propriété est le produit du consentement des parties) et le formalisme du livre foncier (mais elle n'est juridiquement valable que lorsqu'elle est constituée par une autorité judiciaire), ainsi qu'un mode de gestion administratif des mutations concernant les immeubles. Ce n'est donc pas la définition de base du droit de propriété* qui fait la différence (elle est proche de ce qu'on en dit dans les codes des pays latins), mais c'est le mode d'enregistrement ou de "publicité foncière* », autrement dit la présence d'un « cadastre juridique » dont l'outil est le livre foncier. L'existence d'un livre foncier en Allemagne est historique : on l'utilisait dans les villes de la Hanse à la fin du Moyen Âge et il existait au temps du despotisme éclairé en Prusse, et définitivement établi en 1872.

Aujourd'hui, plusieurs pays d'Europe centrale ou septentrionale ont recours au livre foncier : Autriche (depuis 1871), Allemagne, Suisse alémanique, pays scandinaves, Serbie, etc. En raison de l'influence allemande exercée dans l'Empire ottoman dans les années 1880-1914, le droit allemand concerne aussi la Turquie actuelle. De même, l'Alsace et une partie de la Lorraine ayant été annexées à l'Allemagne de 1871 à 1918, ces régions françaises connaissent toujours un régime à part, avec un livre foncier géré par le ministère de la Justice.

Dénommé Grundbuch en allemand, le livre foncier est le registre qui assure la publicité foncière* ou immobilière dans ces pays dans lesquels on a adopté une procédure en deux temps, l'expression du consentement des parties reliant un auteur (terme de droit pour nommer le vendeur) et son acquéreur, puis l'inscription dans un registre ou livre au terme de formalités, inscription sans laquelle il n'y a pas transfert du bien. Ainsi, alors qu'en France on est légalement propriétaire dès qu'on signe l'acte dans l'étude du notaire, en Allemagne, on ne l'est qu'une fois la formalité d'inscription au livre foncier achevée.

Dans ces pays, le lien entre le cadastre et la reconnaissance administrative de la propriété foncière est essentiel. Cependant, on peut définir un gradient dans cette typologie. Il existe des systèmes dans lesquels la fonction juridique est très forte parce que le cadastre est intégré au livre foncier au point que la fonction fiscale est une fonction auxiliaire et non la raison d'être du cadastre (cadastre suisse) et d'autres dans lesquels le 
cadastre vient de la fiscalité et n'a été associé au livre foncier qu'en raison de choix faits à certains moments de l'histoire foncière du pays (Allemagne ; Code civil du Québec de 1994).

\section{L'impact de la colonisation}

La colonisation d'une grande partie du monde est venue perturber cette typologie car la pratique de l'immatriculation des concessions de terres aux colons a, en quelque sorte, unifié les pratiques en les alignant sur le livre foncier. En effet, le registre du système Torrens appliqué dans les colonies anglaises ou le registre d'immatriculation appliqué dans les colonies françaises, malgré les nuances qui les distinguent, optaient pour une définition initiale de la propriété sur une base non consensualiste mais au contraire ressortissant du régime de la concession. Appliquée en milieu colonial, la propriété de l'article 544 du Code civil français a ainsi changé de sens pour devenir un instrument de différenciation et de domination. Ainsi, bien qu'on y applique exactement le même article 544 qu'en France, le contenu et la portée de cette définition ne sont pas les mêmes dans une colonie française qu'en métropole.

La colonisation a permis de poursuivre le mouvement en faveur du livre foncier en lien avec la préoccupation topographique. C'est ce qui explique, par exemple, que dans l'exercice de son mandat en Syrie-Liban, la France ait développé dans ce pays un cadastre à livre foncier fortement appuyé sur une section de topographie qui supposaient, l'un et l'autre, une formation du géomètre et la création d'un enseignement adapté (Kilzi, 2002).

Or, dans le même temps, en métropole, l'évolution de la publicité foncière* était radicalement différente et, en France, on allait s'engager toujours plus dans le sens consensualiste, soit en refusant le livre foncier à la fin du XIX siècle, soit en fixant le régime foncier* avec la loi de 1955. L’une des conséquences était qu'en France, le travail du géomètre se divisait : parce que le géomètre du Cadastre n'avait qu'un rôle fiscal (établir le plan servant d'appui à la définition de l'évaluation foncière pour établir la contribution foncière), il fallait qu'un géomètre-expert soit investi de la délégation de service public pour pouvoir établir les limites des parcelles de propriété et éclairer les parties, dans un bornage consensuel, ou le juge, dans un bornage judiciaire. D'où cette conséquence originale en France qui fait que le plan cadastral du ministère des Finances et le procès-verbal du géomètre-expert présentent d'assez sensibles différences de mesure (car pour établir la contribution fiscale on n'a pas besoin de la précision centimétrique du plan, alors que pour fixer les limites de la propriété cette précision est nécessaire), et ce qui implique que seul le procès-verbal du géomètre-expert fait foi en justice quant aux limites de la propriété.

\section{La complexité des droits fonciers* de l'Europe de l'Est}

Les pays d'Europe centrale qui ont vécu sous un régime socialiste des années 1950 jusqu'au début des années 1990 sont ceux qui possèdent les héritages les plus complexes, et l'histoire agraire la plus heurtée (Maurel, 1994). La raison est qu'ils ont connu, en un siècle, une gamme de formes foncières étonnamment diversifiée : le grand domaine* aristocratique d'ancien régime*, la propriété privée dans le cadre de réformes agraires qui ont partagé les grands domaines, les grandes fermes d'État et les coopératives de production des régimes socialistes, la petite propriété familiale et, enfin, l'entreprise agricole 
actuelle au temps de la reprivatisation qui a fait suite à la chute des régimes communistes. Leurs itinéraires ne se ressemblent pas mais quelques traits communs peuvent être dégagés.

Le plus important est sans doute la force des oppositions idéologiques constatée autour des questions foncières, qui explique que pendant l'époque communiste, la propagande ait ciblé la propriété privée alors qu'elle était assez souvent récente et fragile et alors que c'étaient plutôt les contraintes issues des anciens régimes seigneuriaux qui marquaient encore le plus les comportements ${ }^{4}$. Cette force explique aussi le fait qu'après 1989 on ait estimé urgent de restaurer la propriété privée et que les programmes des partis politiques en aient fait un pivot de leur action.

L'exemple de la Pologne est intéressant à observer. En articulation avec le principe de propriété privée (art. 140 du Code civil d'inspiration latine), cet État a même fait de l'agriculture familiale* un principe constitutionnel (art. 23 de la Constitution de la Pologne de 1997) ${ }^{5}$. Juridiquement, le régime est celui de la propriété privée, dans une conception latine, mais avec la mise en œuvre du livre foncier régi par l'autorité judiciaire, comme dans les pays germaniques. De son récent passé socialiste, ce pays a conservé des spécificités, notamment le régime dit de «l'usufruit perpétuel », qui porte sur l'exploitation des biens de l'État ou ceux des collectivités territoriales, et que l'attributaire peut vendre à son profit, ce qui l'apparente à une forme de propriété. D'ailleurs, depuis une loi de 2005, le titulaire d'un tel droit peut demander sa transformation en droit de propriété*.

Mais les formes mises en œuvre depuis maintenant une vingtaine d'années ne sont pas linéaires (voir Marie-Claude Maurel dans ce volume). La réappropriation privée du bien collectif par la redistribution de parts de propriétés ne se traduit pas par la reconstitution pure et simple d'une couche paysanne attachée à l'agriculture familiale. Des pays ne tranchent pas et instituent un double principe juridique, de façon contradictoire. Par exemple, la constitution de l’Ukraine (1996) fait de la terre à la fois la propriété du peuple ukrainien, tout en garantissant le droit de propriété* privée, et on ne sait pas ce que signifie ce double niveau très ambigu.

On comprend mieux pourquoi et selon quels mécanismes sociaux et fonciers la « grande maille agraire » qui caractérisait les structures de production agricole de l'époque communiste exerce encore une influence et transmet une structure de façon

4. Par exemple, la collectivisation foncière soviétique est d'abord une forme domaniale globale qui impose « la propriété de tout le peuple » ou obscenarodnaja sobstvennost nommée dans les textes de 1917 et 1918. Elle définit un pavage d'unités territoriales, les kolkhozes, qui sont un cadre contraignant : obligation, pour les paysans, d'y travailler, de livrer leur bétail au kolkhoze, suppression du passeport aux kolkhoziens en décembre 1932 pour lutter contre l'exode, d'appliquer la planification économique. On voit ainsi comment un régime socialiste a pu reprendre, en la renommant, une structure communautaire ancienne (obscina) mais pour un tout autre contenu (Marxer, 2003). Cependant, on a fait du producteur privé (le koulak.) le bouc émissaire des difficultés de l'agriculture soviétique, alors qu'il ne représentait pas le type principal qu'on rencontrait dans les campagnes, loin de là. Dès lors, présenter la révolution soviétique comme une entreprise d'abolition de la propriété privée était donc politiquement efficace, mais en grande partie une ambiguïté puisque la propriété privée personnelle n'était pas, et de loin, la forme dominante en Russie au début du $\mathrm{xx}^{\mathrm{e}}$ siècle. Sur le plan juridique, ce qu'a fait le régime socialiste ce fut de conserver la domanialité : il n'y a pas eu de réforme agraire et de partage de la terre avec propriété privée, mais maintien des formes communautaires, dans un autre habillage et dans un autre objectif social. La situation est évidemment différente dans les pays de l'Europe de l'Est.

5. Même chose en Roumanie, avant la période communiste, où il s'agissait de protéger les petites exploitations issues du démembrement des anciens domaines féodaux. 
diachronique (Maurel, 2012). Ces pays sont entrés dans une transition complexe et s'avèrent ainsi des candidats à l'agriculture de firme*, tout en ayant été des partenaires très spécifiques dans la définition de la politique agricole commune*.

\section{Différences conceptuelles}

\section{Formes latines et formes anglo-saxonnes}

Le fait est bien connu mais toujours difficile à expliquer et à prendre en compte : sous la même expression de "droits de propriété », on ne met pas du tout le même sens selon qu'on est dans le droit latin et germanique ("romano-germanique »), ou le droit anglo-saxon. La raison est que, dans le premier, on raisonne sur la base d'une institution de la propriété ${ }^{6}$ pour savoir ce à quoi elle donne droit, alors que, dans le second, on raisonne économiquement sur la base de savoir ce que la propriété permet de faire. Ainsi les property rights anglo-saxons sont un concept de l'économie et non du droit, une posture dans laquelle il est moins important de savoir le contenu juridique du droit en question que de chercher, dans le développement de la jurisprudence, les différentes façons de générer des solutions. Voilà pourquoi dans un cas on va chez le notaire, dans l'autre chez le lanyer, celui-ci étant autant avocat que notaire.

Le point essentiel à retenir est le suivant : l'articulation entre les systèmes de droit foncier et l'économie fonctionne mieux dans le système foncier anglo-saxon, parce que celui-ci conserve cette structure juridique de superposition des droits héritée de l'histoire anglaise, parce qu'il préfère la jurisprudence au Code, parce qu'il se passe très bien de l'État, et sait trouver des solutions pour contourner les obstacles. Par exemple, avec le trust, les Anglais disposent d'un mécanisme de déplacement de l'intérêt, du bien vers la valeur du bien, du bénéficiaire vers le trustee, nettement plus souple que les formules du mandat, du pouvoir, du fidéicommis telles qu'elles se pratiquent en droit latin.

Les théoriciens économistes savent exploiter ces différences. Mais la tendance dans la première moitié $\mathrm{du} \mathrm{xx}^{\mathrm{e}}$ siècle a été d'infléchir la voie libérale ou ultralibérale. Par exemple, pour l'économiste Charles R. Noyes (1936), qu'on remet aujourd'hui à l'honneur, le droit contemporain puise à deux sources, le droit romain et le droit féodal anglais $\mathrm{du} \mathrm{xI}^{\mathrm{e}}$ siècle. Traitant des deux sources, il oppose un système dérivé et linéaire (lineal system), de type féodal, issu de la common law, et un système direct et collatéral, de type romain ou romaniste, issu du droit continental.

Le système légal romain de propriété, conformément aux relations externes, peut être caractérisé comme étant le système allodial ou collatéral. C'est la propriété selon le droit absolu et originel par des unités indépendantes et coégales ; le caractère des intérêts étant le même. Le système légal anglais, plus conforme aux relations internes [qu'on ne l'est] à Rome, peut être nommé le système féodal ou linéaire. C'est la propriété, sauf au sommet, [conçue] selon un droit dépendant et sans doute dérivé, par des unités successives et hiérarchiques, selon les degrés variables d'ascendance ; les intérêts [sur le sol] étant mutuellement exclusifs (Noyes, 1936, p. 222 ; ma traduction).

6. Comme je le fais d'ailleurs moi-même dans cet article où je n'échappe pas à ma culture historienne et où je ne néglige pas la réflexion sur l'établissement des normes. 
Telle quelle, cette typologie peut séduire les juristes car elle renforce l'opposition entre common law et droit civil qui leur est familière. Mais, elle est historiquement biaisée car ce que Noyes appelle « romain » n'est que la représentation que les modernes se font du droit romain (leur récit* recomposé du droit romain), et pas la réalité juridique romaine. Ainsi, l'étude de Charles R. Noyes nous fait comprendre les origines savantes des idées des économistes sur la nécessité du titre pour consolider les droits. On doit en effet se souvenir que Noyes réclamait une codification, défendait une «institution de la propriété » (titre de son livre), une systématisation et une cristallisation des règles de droit en un corpus unique « pour échapper aux mystères et aux incertitudes de la loi non écrite ou de la jurisprudence $»^{7}$. Il plaçait donc la typologie au service du développement du formalisme, dans une approche plus latine qu'anglo-saxonne. Mais ensuite, il exposait explicitement le lien de filiation existant entre l'architecture des intérêts dans un trust moderne et celle des estates dans le système médiéval anglo-saxon. Il écrivait :

Le système moderne des intérêts dominants dans le trust et le fonds d'entreprise (corporate fund) a déjà été mis en parallèle avec le système médiéval des estates dans la terre (estates in land). Il est variable, flexible, progressif (Noyes, 1936, p. 527 ; ma traduction).

La voie préconisée par Noyes n'a pas été suivie, et la notion de droits de propriété en économie du droit ${ }^{8}$, pensés comme droits économiques, a acquis une grande importance. Ainsi, et c'est une insuffisance permanente des discours critiques, il faut entendre cette notion de droits de propriété non pas comme une théorie du titrement de la propriété - chez les économistes c'est un aspect secondaire -, mais comme une théorie des droits d'usage exclusifs et librement transférables existant autour des biens. Les property rights sont des «droits d'accès à », et sont différentes de l'ownership, qui est l'appropriation privée ${ }^{9}$. Les property rights sont des droits ouverts, ce qui signifie qu'ils sont soumis aux mécanismes du marché, ce qui explique leur répartition et leur agencement. Lorsque l'expression est employée par les économistes, elle n'a pas de sens juridique (Galey, 2007, p. 10).

Ces théories économiques font un lien entre diverses réalités ou représentations du monde actuel : la peur de l'accroissement démographique qui conduit à une forme de néomalthusianisme ; la perception de la finitude du monde et de ses ressources, d'où la nécessité, selon certains, d'achever l'occupation et la mise en exploitation de la surface terrestre ; la nécessité de le faire dans le cadre de l'économie de marché car les sociétés traditionnelles seraient des sociétés incapables ; la nécessité de formaliser l'appropriation par le titre afin d'en faire un instrument économique, c'est-à-dire de calquer le juridique sur l'économique. Sur le terrain du foncier*, il s'agit de thèses à la base desquelles on trouve quelquefois le modèle des fronts pionniers, censé rendre compte de l'avancée de l'occupation.

Telle quelle, cette théorie est un des plus puissants lieux communs actuels, par sa diffusion dans les sphères dirigeantes mondiales.

7. Noyes, 1936, p. 22, cité par Soto, 2005, p. 202-203 qui exploite cette dimension du travail de Charles R. Noyes jusqu’à reprendre le terme même de mystère, puisqu'il en a fait le titre de son essai.

8. Law and Economics en anglais, discipline formalisée dans les années 1960.

9. Et il existe une difficulté pour traduire en français ces deux notions (property rights et ownership) puisqu'on parle de droit de propriété dans les deux cas. 


\section{Construire l'espace économique sans politique et sans droit}

La force actuelle de la théorie économique des "droits de propriété » s'explique aussi par une tendance de fond de la pensée économique depuis plus de deux siècles : construire l'espace économique comme un espace affranchi de toute influence politique, de toute lutte pour la définition des frontières. Voulant échapper aux innombrables guerres et incohérences dont l'Europe avait été le témoin de la fin du Moyen Âge au début du XIX ${ }^{\mathrm{e}}$ siècle, les économistes ont élaboré des théories cherchant à s'affranchir de tout rapport avec la politique et ses manifestations.

Pour cela, ils ont théorisé un rapport morphofonctionnel entre l'individu et le sol, qui passe par une très forte géométrisation de l'espace. Pour la science économique naissante des $\mathrm{XVIII}^{\mathrm{e}}$ et $\mathrm{XIX}^{\mathrm{e}}$ siècles, le principe serait le suivant : les divisions spatiales doivent être le produit de l'économie, pas de la politique. Quelle que soit l'option choisie on va assister alors à une «naturalisation » de l'espace économique, c'est-à-dire à la recherche de lois naturelles et intemporelles, qui régiraient l'espace économique et qui échapperaient de plus en plus à la contingence historique. Ce sera le rôle d'une nouvelle discipline la Nationalökonomie (l'économie politique) ${ }^{10}$, que d'offrir les règles permettant de se défaire de l'espace politique au profit de l'espace de marché.

La genèse de ces idées est utilitariste à base de modèles gravitaires (Garner, 2005). La thèse centrale de l'utilitarisme proclame la logique égoïste du calcul des plaisirs, des peines, et des intérêts, mesurable à partir d'une typologie de critères : durée, intensité, certitude, proximité, étendue, fécondité, pureté. Comme dans l'utopie, on fait le lien entre bonheur public et forme géométrique, entre la fonction et la forme. Le théoricien Bentham pense même que la norme utilitariste est une norme unique et universelle, valant pour toutes les autres dimensions de l'existence sociale. Ce serait donc une loi naturelle, une espèce d'invariant anthropostructuraliste. La norme serait transférable au droit naturel (dont elle constitue la base), ainsi qu'au territoire. Mieux, elle procéderait de la nature. L'utilitarisme étend ainsi la notion de lien contractuel à des choses très diverses, dont le contrat social et le contrat territorial, posant les bases d'un lien morphofonctionnel entre l'individu et la parcelle. Pour que le calcul de l'utilité collective soit faisable, Bentham ajoute qu'il faut que tous les individus soient tenus pour égaux.

On voit donc que ce qui justifie le passage de telles théories sociales dans des modèles gravitaires d'explication de la répartition des choses, c'est un ensemble de dispositifs conceptuels et normatifs qui ont pour nom : la mesure; l'égale répartition des choses ; l'égalité civique ; l'isotropie globale de l'espace et des sociétés ; la géométrie. On voit donc aussi ce qui fait le fond des théories anglo-saxonnes libérales des XVIII et $\mathrm{XIX}^{\mathrm{e}}$ siècles : ce n'est pas le rejet de la norme, c'est le fait que la norme ne doit pas être instituée par le politique mais doit venir de lois naturelles. De la sorte, cette pensée géométrique rejoint le combat des élites aristocratiques pour échapper à l'emprise de l'État et de sa fiscalité, si vif en Angleterre aux XVII ${ }^{\mathrm{e}}$-XIX ${ }^{\mathrm{e}}$ siècles, lors du mouvement des enclosures; ou, à un moindre niveau, en France au moment de la Restauration de la royauté, lorsque les ultraroyalistes tentent de revenir sur les acquis fonciers de la Révolution et de restreindre la portée du cadastre, en cours de réalisation (Vivier, 2008).

10. Mais le nom même de cette théorie en allemand est une singulière contradiction avec son contenu, au moins par rapport au sens que nous donnons au terme «national»! 


\section{Pourquoi y a-t-il crise de la propriété ?}

Le diagnostic de la crise de la propriété est souvent exprimé : on assisterait à sa fin. Avant de s'interroger sur le bien-fondé de cette affirmation, il est utile de savoir dans quel contexte émerge cette notion de crise de l'idée de propriété. Car la crise de la propriété est liée à la crise de la Modernité, la propriété ayant toujours été, selon de nombreux auteurs, le principal pilier de la Modernité occidentale.

\section{Croissance du mouvement d'hostilité aux normes et à l'État}

La volonté d'aboutir à un espace économique seulement régi par les lois du marché va de pair avec le rejet de tous les horizons normatifs hérités de l'histoire récente et des disciplines qui l'ont porté ${ }^{11}$. Le refus des idéologies, dans une ambiance de « fin de l'histoire », apparait ainsi comme une prétention placée au centre de l'idéologie libérale et néolibérale des dernières décennies. Elle se traduit par un affaiblissement marqué de l'intérêt pour l'État, qui peut aller jusqu'à une franche hostilité. Mais deux aspects différents sont en jeu, qui interdisent toute appréciation simpliste.

D’un côté, on pointe du doigt la centralisation et ses excès, au profit d'une conception décentralisée ou subsidiaire, différente et plus démocratique. Mais d'un autre côté, on fait subir aux institutions, et d'abord à l'État moderne, la réduction à l'état d'entreprise économique et donc la soumission aux évaluations ambiguës de l'économie et de l'économétrie ${ }^{12}$. Ce mouvement réducteur, précisément, n’a pas de nom évident en français, car, jusqu'à présent, il est plutôt étranger à la culture latine, alors qu'il est aisément qualifié en anglais par l'adjectif corporate*. Le terme « entrepreneurial » par lequel on le traduit semble, en effet, plus banal en français que ne l'est corporate en anglais, et il n'a pas ce sens réducteur parce qu'en français, on ne l'associe pas spontanément à la thèse correspondante. Car ce qui est en jeu, c'est le remplacement de notions telles que la sphère publique, l'État, le droit, la souveraineté, et les institutions qui en découlent, dont la propriété, par des notions telles que la gouvernance, la coproduction publique et privée des normes, la délégation du pouvoir d'évaluer à des agences non élues, etc.

Existera-t-il un jour prochain un ordre juridique hors de la protection que tentent d'assurer les États ? C'est d'abord aux théoriciens de la logique libérale de mondialisation* qu'il faut poser la question, mais sans exonérer les défenseurs des logiques alternatives - notamment de la logique des communs - de leur part de responsabilité dans cette évolution, car la promotion du « commun » au lieu de la défense du «public » ne manque pas de soulever des questions similaires et contribue à cette même érosion des structures étatiques et de souveraineté héritées du XIX et du $\mathrm{Xx}^{\mathrm{e}}$ siècles. En matière foncière, quelles formes de garantie entend-on promouvoir, si on réduit ou supprime

11. Il serait intéressant de mettre en parallèle la crise de la propriété avec la crise des disciplines. Sans être long, l'enjeu est le suivant. Dans la question de la propriété, on hésite entre des formes définies au XIX ${ }^{e}$ siècle et des formes nouvelles de recomposition des usages ou des utilités (ce que je développerai plus avant dans le texte) ; dans les disciplines on hésite de même entre le maintien d'une division positiviste du savoir, héritée du $\mathrm{XIX}^{\mathrm{e}}$ et du Xx ${ }^{\mathrm{e}}$ siècles et la création de nouvelles (et innombrables) disciplines d'hybridation, en se demandant d'ailleurs pourquoi toutes ces nouveautés ne réussissent pas à proposer un nouvel ordre épistémologique.

12. Voir l'article d'Hubert Cochet dans ce même volume pour d'intéressantes considérations sur l'évaluation. 
la garantie publique au profit d'une évaluation économique libérale, ou d'une définition exclusivement communautaire?

L'idée que le droit pourrait se passer de l'État est liée à la mondialisation* et à la croyance en une société capable de s'autoréguler. C'est le credo libéral depuis Locke et Smith. La nouvelle norme de cet espace mondialisé serait la concurrence. Or le droit de la concurrence « est impuissant à fonder un ordre juridique, car il ne connait que la circulation des produits et ignore le sort des hommes » (Supiot, 2005). Dans un espace aplati (c'est une allusion à la thèse de Thomas L. Friedman, 2006), dans une vision idéalisée de la mise en réseau, la thèse libérale imagine qu'une société de contrat pourrait être une société sans conflits. Reposant sur le seul consensualisme, et celui-ci dessinant la réalité par ses incessantes recompositions, on en viendrait à penser que parce que le contrat est en théorie un acte libre entre deux parties, le droit basé sur le contrat pourrait faire l'économie de la prise en compte de la dimension conflictuelle du monde social. Et on laisserait à l'État la charge de se débarrasser de l'étroitesse des catégories sociales héritées. Or ce n'est pas inventer de nouvelles normes que de laisser le jeu se faire entre le corporate* world et le corporatisme catégoriel.

\section{Le sentiment de fin de cycle juridique}

La crise de la propriété rejoint une idée plus générale, celle du sentiment que nous serions à la fin d'un cycle juridique ou d'un monde, celui de la science du droit. Tel est l'avis, par exemple, de Frédéric Zénati-Castaing, un éminent civiliste* français. Nous serions non seulement à la fin du droit, mais aussi à la fin de la science du droit, c'est-àdire de cette construction intellectuelle amorcée au $\mathrm{XI}^{\mathrm{e}}$ siècle au moment de la redécouverte (ou « réception ») du droit romain par l'Occident. Ainsi, ce qui disparait, c'est l'idée qu'on puisse voir et concevoir le droit à l'aide d'abstractions. Il ne resterait plus que la réalité prosaïque. Les modèles propriétaristes* du XIX et du $\mathrm{XX}^{\mathrm{e}}$ siècles auraient vécu. On retrouve le même sentiment chez le juriste italien Aldo Schiavone (2008), qui annonce la fin de l'influence du droit romain, celui qui a fait de la propriété et du contrat la base des élaborations juridiques et sociales pendant des siècles.

Un monde qu'on nous dit sombrer. Or, ce que constatent ces juristes, c'est la fin de la construction du droit comme sphère d'étude indépendante des sociétés, ce qui est une des inventions épistémologiques les plus curieuses et les plus durables de l'Occident ${ }^{13}$. Mais une autre écriture du droit est possible, plus historicisée, plus en prise avec les réalités. Peut-être n'est-ce pas la science du droit qui meurt mais peut-être est-ce le splendide isolement des juristes qui prend fin?

Cependant, le changement des représentations est manifeste. Quelle est la profondeur de cette mutation? Que devient, dans cette nouvelle représentation, la propriété ? Celle-ci meurt-elle en même temps que le droit, parce qu'elle en serait une des principales émanations?

À l'époque de la définition de la propriété par le Code civil de 1804, l'exclusivité et son caractère absolu étaient des progrès par rapport à la situation d'ancien régime*.

13. Dès l'Antiquité, les jurisconsultes ont eu la nette conscience de l'autonomie de leur monde intellectuel. Mais cela s'est renforcé avec l'étude du droit romain par la science juridique allemande au xix ${ }^{e}$ siècle. La métaphore employée pour parler du droit romain, c'est-à-dire le droit civil, était horlogère. Le droit était une mécanique insensible aux faits sociaux. Avant l'économie, le droit entendait déjà s'affranchir de la politique. 
Quand on disait que la propriété était exclusive et absolue, on voulait faire comprendre le regroupement de toutes les utilités dans la main du propriétaire, afin d'éviter la situation de propriété divisée entre (domaine) éminent et utile*, entre seigneur et tenancier. Ainsi, techniquement, la propriété est exclusive parce qu'elle réunit dans la même personne les prérogatives du propriétaire ; elle est absolue parce que la propriété est un droit illimité par opposition aux droits incorporels qui sont relatifs ou limités et parce qu'elle est source d'opposabilité (Zénati-Castaing et Revet, 2008, p. 313 sq.). C'est aussi ce caractère absolu et illimité qui fait qu'en droit français, la propriété ne se prescrit pas par le non-usage, alors que dans d'autres systèmes fonciers, l'obligation de mise en valeur est décisive et que la négligence peut faire perdre la propriété.

Or aujourd'hui, ces deux aspects sont dévalorisés et relativisés et ce qui était « progrès » il y a deux siècles, semble être devenu une faute irrémédiable. Au mieux on soumet la propriété à de nombreuses restrictions, ou on cherche à la diviser ; au pire, on la réprouve et on la rejette. Ainsi, il y a eu inversion des représentations.

Cela va-t-il jusqu'au constat de décès du droit de propriété*, comme certains juristes le prétendent? Dans un pays comme la France où la propriété est un principe constitutionnel, c’est (encore) difficilement soutenable car la propriété est « un droit essentiel dans la constitution de la personnalité juridique, figure centrale de la modernité sociale et juridique » (Zenati-Castaing et Revet, 2008, p. 350).

\section{Les thèses libérales et les alternatives}

Le panorama qui suit expose de nombreuses approches théoriques ou méthodologiques prêtant largement le flanc à la controverse, les unes favorables à l'extension de la propriété, les alternatives à ce modèle. Cependant, les démarches alternatives n'ont pas encore inspiré de nouvelles pratiques et modalités de gestion du foncier* ou ne les ont modifiées qu’à la marge.

\section{Des théories libérales plaident pour la formalisation de la propriété}

\section{Thèse de la tragédie des communs de Garret Hardin}

Depuis le texte de Garrett Hardin sur la "tragédie des biens communs », publié en 1968, diverses idées forment le socle d'une vision fortement régulatrice de l'avenir, sur la base de la contrainte mutuellement convenue entre les gens. Ce biogénéticien est intervenu sur le terrain économique pour défendre l'idée que le laisser-faire en matière de biens communs est la source de tous les dangers et qu'il faut trouver des systèmes de régulation et de sélection de l'accès aux biens. Selon lui, lorsqu'une ressource est en libre accès, chaque utilisateur est conduit spontanément à y puiser sans limite, poussant à sa disparition. Dans un pâturage en libre accès, chaque éleveur cherche à accroittre son troupeau puisque, de toute façon, le prix à payer est quasi nul par rapport au bénéfice immédiat obtenu. Mais, au terme de ce processus, tous les éleveurs sont perdants par épuisement rapide de la ressource. Jean-Marie Harribey (2011, p. 101) commente :

On relève ici une parenté de cette «tragédie » avec la thèse de la surpopulation que Malthus avait énoncée à la fin du XVIII ${ }^{\mathrm{e}}$ siècle. Selon Hardin, il n'y a que 
trois solutions à cette «tragédie » : la limitation de la population pour stopper la surconsommation, la nationalisation ou la privatisation. Émise à la veille du grand mouvement de dérégulation et de déréglementation de l'économie mondiale, on comprend que la troisième voie fut exploitée à fond pour justifier le recul de l'intervention publique.

Garrett Hardin cite diverses solutions pour limiter l'accès à la ressource : la vente aux enchères, le mérite, le hasard (loterie), le premier arrivé, et il note que la propriété privée a joué ce rôle en ce qui concerne les biens immobiliers. Une autre idée défendue par le même auteur est que la moralité d'un acte d'appropriation de l'espace est fonction de l'état du système au moment de l'acte. Autrement dit, la même prédation est tolérable dans un espace vide, mais insupportable dans un espace surpeuplé. Ensuite, il pense qu'autant on peut légiférer en matière d'excès (ex. la prohibition), autant on a du mal à le faire en matière de modération, bien que nous ayons la nécessité d'en passer par «l'invention de rétroactions coercitives ». On sait aussi que, dans son article, il s'en prend à l'image pour nier son caractère représentatif, si elle n'est pas accompagnée des mots ou des discours qui permettent de passer de l'instantané et du localisé au système total dans lequel l'image peut prendre sens.

Le dilemme que pose Hardin, sur la base du constat de l'augmentation de la population, est entre la ruine totale, si on laisse faire, et l'injustice que représente un accès limité aux biens communs, solution imparfaite mais préférable.

\section{Thèse de la défaillance du droit chez Hernando de Soto}

Hernando de Soto (2005 pour la traduction française) nomme « défaillance du droit» la béance qui se constate entre les situations de fait de l'occupation du sol et les cadres légaux, notamment en matière de propriété. C'est un fait incontestable : dans les pays en développement et dans les anciens pays communistes, le droit laisse de côté la majeure partie des terres, jusqu'à $80 \%$. Pour expliquer cette situation et avancer des solutions, cet auteur brosse un récit* original : le droit de propriété* fonctionne en Occident alors qu'il ne fonctionne pas partout ailleurs. Cette thèse est le cœur de ce qu'il nomme le «mystère du capital », titre de son livre devenu classique.

Hernando de Soto s'inspire directement des idées de Charles Reynold Noyes qui voulait échapper à l'informalité et à ses mystères. Il faut donc, commente Hernando de Soto, créer un régime unique de propriété, « en découvrant comment et pourquoi les conventions locales fonctionnent et à quel point elles sont solides » (p. 201). Cela passe par la critique de quelques idées fausses :

1. On a cru que la propriété n'existait pas pour éviter de payer des impôts ; mais les coûts de l'économie souterraine sont importants ;

2. On a soutenu que l'arpentage et l'enregistrement cadastral ne seraient pas les conditions suffisantes pour passer au niveau légal ;

3. Qu'il ne suffirait pas de mettre en application un droit de la propriété obligatoire pour que cela marche ;

4. Qu'on ne pourrait pas ignorer les arrangements informels existants ;

5. Qu'on ne pourrait pas modifier vraiment ces situations sans signe politique fort.

Hernando de Soto prend alors appui sur l'analyse de Marx mais en l'inversant. Celui-ci avait noté combien le capital est quelque chose d'autre que les formes matérielles de l'économie, l'argent, les matières premières, etc. Sur cette même base, Hernando de Soto observe que la propriété formelle peut, par le biais de représentations, 
conférer aux ressources économiques des fonctions supplémentaires et un surplus de valeur. Il pense donc que la propriété est la représentation qui peut « faire apparaitre le potentiel économique des biens » (p. 264). Selon lui, Marx est donc dépassé en ce sens que le capital potentiel, nommé « capital dormant ", n'est plus l'apanage d'un petit nombre de capitalistes, mais qu'il est présent chez de nombreux petits entrepreneurs extralégaux dans les pays en développement. La propriété est donc ce qui rend le capital « intelligible », en ce qu'elle fait entrer les biens dans le marché et en ce qu'elle fournit aux populations le raisonnement qui permet d'extraire du capital des ressources, capital que son étude chiffre, dans une précision un peu étonnante, à... 9300 milliards de dollars.

La difficulté de la thèse de Hernando de Soto vient du fait qu'elle ne s'applique qu'à certains types d'espace. Aujourd'hui, on assiste plus souvent à des concessions massives de terres agissant sur des terres prétendues vides et qui ne le sont pas toujours en fait. On est donc à l'opposé de la reconnaissance de l'informalité des occupations existantes puisqu'on nie même qu'il y ait des occupants. Il faudrait donc suggérer à Hernando de Soto d'écrire un chapitre nouveau de son livre dans lequel il prendrait en compte ces situations nouvelles et de dire en quoi elles infléchissent son modèle, ou mieux de se demander pourquoi on ne respecte pas ses propres préconisations, alors qu'on donne un grand poids intellectuel à cet auteur.

\section{L'entreprise n'a pas toujours besoin de la «propriété foncière »}

On pourrait être conduit à faire une analyse inexacte de la réalité si on posait comme attendu que la règle libérale est de diffuser partout la forme de la propriété foncière afin de pouvoir immédiatement la mobiliser dans les circuits économiques. Le courant libéral ne se résume pas à cela et dans diverses situations très contemporaines, c'est vers d'autres voies qu'on s'oriente. En effet, dans une économie libérale qui a changé, pourquoi perdre son temps à formaliser la propriété foncière quand le rapport au sol n'est plus la base principale et que ce qui compte c'est d'identifier le segment d'une chaîne de valeur qu'il est utile de contrôler, et lui seul ? Différentes formes actuelles récemment développées en témoignent.

Que signifie être propriétaire quand on peut se retrouver en situation de rapport ou de contrat inversé (voir le métayage inversé dont parle Hubert Cochet, dans ce livre) ? On désigne, dans la littérature anglo-saxonne, par l'expression de reverse tenancy, des situations dans lesquelles des petits propriétaires cèdent en faire-valoir indirect une partie ou la totalité de leurs disponibilités foncières à de (plus) grands propriétaires ou à des entrepreneurs agricoles disposant du capital d'exploitation, capables de mobiliser les financements, les équipements, de maîtriser le fonctionnement des marchés. C'est la différence des statuts sociaux qui fait l'originalité du rapport puisque le cédant est un petit propriétaire, le preneur ou tenancier un gros ou un très gros exploitant, ou encore une entreprise. Jean-Philippe Colin $(2013$; 2014), propose de traduire l'expression anglaise par «tenure inversée » : c'est une forme de concentration foncière par l'exploitation, qui passe par des contrats de location ou par des contrats de métayage (reverse sharecropping), et non par une concentration de la propriété. Elle n’est pas nouvelle mais elle tend à devenir une pratique répandue.

Un autre exemple de ces situations où l'entreprise n'a pas besoin de la propriété formelle pour agir se rencontre avec les concessions massives de terres dans les pays en développement ou dans les pays d'Europe les plus ouverts à ce mode. Comme la 
démonstration en a été faite (Chouquer, 2012), on constate que l'investissement passe par des baux emphytéotiques, et que, pour les entreprises, ce qui importe ce n'est pas d'être propriétaire mais de pouvoir négocier en bloc la location des terres, sans passer par de longues négociations avec les populations locales. Il a ainsi été démontré que c'est la situation juridique de domanialité qui, en faisant des occupants actuels des occupants sans titre, simplement tolérés, permet de les déplacer pour libérer les terres au service de la concession. Ensuite, l'État immatricule les terres à son nom avant de les louer aux entreprises.

\section{Des communs historiques aux communs actuels : l'économie du partage}

Le succès actuel de la réflexion sur les communs en anthropologie et en science politique, ne doit pas faire oublier l'antériorité et l'intérêt d'une analyse des « communs historiques » par les historiens et les géographes (ex. Demélas et Vivier, 2003) et par les juristes (Gau-Cabée, 2006). Ces différents travaux offrent une base pour travailler car ils ont établi des typologies juridiques et sociales argumentées et proposé un cadre de réflexion comparatiste pour toute l'Europe. Les héritages ne sont pas tous les mêmes et Nadine Vivier a dégagé une carte européenne dans laquelle elle a distingué trois zones différentes aux XVIII $^{\mathrm{e}}$ et XIX ${ }^{\mathrm{e}}$ siècles : une zone nordique dans laquelle la question des communaux est déterminée par la seigneurie (Bretagne, Angleterre, Prusse) et où le partage des communaux s'est fait en proportion des propriétés et favorise les propriétaires, provoquant le départ des journaliers (Angleterre, Allemagne du Nord, Danemark) ; une zone médiane ou méridienne de l'Europe dans laquelle les communautés, en tant que corps juridiques, détiennent les terres et en régissent l'utilisation (de l'Allemagne du Nord à l'Italie du Nord), et qui peut partiellement recouper la zone de partage égal entre les ménages (Belgique, France) ; une zone méridionale dans laquelle le rôle de l'État central persiste et où est privilégiée la propriété institutionnelle des communes (en gros l'ancienne Europe des monarchies des Bourbons : péninsule Ibérique, France du Sud, Italie centrale et méridionale) et où la privatisation passe par la vente.

Mais aujourd'hui, ces communs fonciers historiques sont débordés par l'extension de la notion aux biens communs mondiaux et par l'apparition de « nouveaux communs » (internet, les logiciels libres, l'open science, etc.). La propriété en tant que concept s'en trouve fortement influencée. D'innombrables travaux tentent d'ériger en principe la notion de " commun », afin d'en faire le terme central d'une alternative politique pour le $\mathrm{xxI}^{\mathrm{e}}$ siècle, et de créer un dénominateur commun pour toute une série de luttes qui souhaitent allier la contestation du modèle libéral ou néolibéral à l'écologie politique. L'opération en cours consiste à faire valoir que les communs historiques sont une forme de propriété oubliée qui a été écrasée par le dogme du propriétarisme ( impasse du droit actuel », « crise de l'idéologie propriétaire et de l'exclusivisme »), et que, dans le même temps, les nouvelles conditions économiques et sociales existant dans le monde imposent un élargissement de la notion, et obligent à « repenser les biens communs » (Parange et Saint-Victor, 2014). Pour certains, il s'agit d'anticiper la " révolution du Xxi ${ }^{\mathrm{e}}$ siècle » (Dardot et Laval, 2014), ou d'offrir un « fort espoir de transformation sociale » (Coriat, 2015), bref, de revenir à du politique.

Exprimée en termes juridiques, la question des communs tourne autour d'un renouvellement de l'accès aux diverses utilités offertes par les milieux et par les ressources dans 
le cadre de la mondialisation*. Il s'agit de repenser les notions juridiques de destination et d'affectation et de dégager une option ou un troisième terme, qui ne soit ni le public, ni le privé, mais le commun.

La théorie des communs comporte alors une critique des limites de la catégorie du public (domaine public, service public, bien public mondial). La base intellectuelle est la suivante : pour qu'un bien ou un service soit public, il faut qu'il soit institué et ensuite garanti par une autorité. Jusqu'ici c'était l'État qui assurait cette fonction. Avec la mondialisation* et l'affaiblissement de l'État on voit bien que cela devient plus difficile. L'exemple de la catégorie des biens publics mondiaux en est l'exemple, puisque personne n'est capable d'en définir et surtout d'en protéger le régime juridique éventuel. Les théoriciens proposent de surmonter la difficulté par «le contournement des propriétaires ", et par la mise en place d'une théorie de l'accès qui prenne modèle, par exemple, sur le régime des monuments historiques, lesquels peuvent être propriété privée, mais soumis à des restrictions imposées par leur caractère patrimonial commun (Rochfeld, 2015). Il s'agit de réaliser l'objectif par un élargissement des titulaires en renversant le paradigme de la propriété privée et s'appuyant sur le bundle of rights, ou faisceau d'utilités, celles-ci pouvant être ouvertes et dissociées. Et dans la gamme des possibles, il s'agit de dire qui sera «mis en saisine* , qui sera « investi » de telle ou telle utilité, ce qui explique le recours de certains juristes aux notions du droit médiéval ou « féodal ».

\section{La thèse d'Elinor Ostrom}

Cette économiste refuse de se situer dans le dilemme entre collectivisme et philosophie libérale individualiste. Dans la seconde moitié du $\mathrm{xx}^{\mathrm{e}}$ siècle, le modèle standard du libéralisme avait été critiqué pour ses échecs. Des théoriciens dits néoclassiques ont alors proposé des modifications théoriques, par exemple en introduisant des hypothèses d'information et de concurrence imparfaites et en relâchant l'hypothèse de rationalité parfaite, et tout en prenant également en compte le rôle des institutions. Pour ces raisons, on a nommé « néo-institutionnaliste » ce nouveau courant de la théorie économique.

Elinor Ostrom accepte l'essentiel de ce cadre théorique rénové. Reprenant la discussion là où Garrett Hardin l'avait laissée, Elinor Ostrom veut « contribuer au développement d'une théorie valide au plan empirique des formes d'auto-organisation et d'autogouvernance de l'action collective » (Ostrom, 2010, p. 40), de telle sorte que «les appropriateurs ${ }^{14}$ adoptent des stratégies coordonnées » (ibid., p. 54). C'est une façon de dire qu'on n'est pas condamné, comme on l'est dans la théorie de Garrett Hardin, à une régulation sélective pour éviter la tragédie. Pour dire ce qui est commun, Elinor Ostrom ne se focalise pas, en effet, sur la nature des biens, mais préfère observer, au contraire, le cadre institutionnel et réglementaire qui fait que les biens deviennent ou sont considérés ou même mieux sont institués comme biens communs. Divers terrains d'étude lui ont permis d'en arriver là. Ce sont à chaque fois des communautés de taille limitée : il importe que chaque membre de la communauté ait une connaissance préalable complète des règles d'accès à la ressource. Ainsi, le fonctionnement est optimal car à une régulation dont on ne comprendrait pas la logique (par exemple, la « main invisible du marché » de la théorie libérale depuis Adam Smith), se substitue une régulation par le jeu conscient et accepté des accords et des coordinations. On comprend alors pourquoi

14. Le terme tel qu’il est traduit est un néologisme ; en français, on traduirait par une périphrase : «ceux qui s'approprient». 
Elinor Ostrom en vient à considérer qu'une approche en commun de la ressource est meilleure qu'une définition par la notion de «public ». Elle écrit :

Une personne qui contribue à la fourniture d'un bien purement public ne se soucie pas vraiment de qui d'autre l'utilise, ou quand et où, du moment qu'un nombre suffisant d'autres individus partagent le coût de la fourniture. Une personne qui contribue aux activités de fourniture d'une ressource commune est très attentive au nombre de personnes qui l'utilisent et aux conditions de leur utilisation, même si ces autres personnes contribuent toutes à sa fourniture (Ostrom, 2010, p. 8, n. 5).

Sur cet aspect précis, c'est le même raisonnement que celui de Garret Hardin : en limitant le nombre des personnes qui accèdent aux ressources, il y a moins de problèmes que si on ouvre à tous ! Mais à la différence près que la délibération collective permanente interdit les appropriations massives, comme l'ont été les enclosures en Angleterre, ou en Prusse au Xix ${ }^{\mathrm{e}}$ siècle. Il n'est pas difficile de relever que cette thèse concerne les sociétés qui, bien que contemporaines de la société de marché, conservent une structure coutumière de type ancien ayant peu de relations avec cette société et donc encore relativement protégée des transformations que ne manquerait pas de provoquer le contact direct avec le marché, en raison des différences de structure et de l'incommensurabilité des échelles (Harribey, 2011).

\section{Les avancées du droit de l'environnement}

Quel serait le droit du sol dans un droit de l'environnement qui serait lui-même conçu comme un véritable droit de la nature ? Rassemblant de nombreux travaux de juristes spécialisés sur ce thème, François Ost a suggéré de faire du patrimoine un statut juridique pour le milieu. Bousculant le rapport cartésien du sujet et de l'objet, exploitant les possibilités du systémisme (" tout fait système dans la nature »), intégrant la complexité et la soutenabilité, il lui a semblé que le thème du patrimoine rassemblait tous les défis.

Pour répondre à autant de défis, la science du droit ne dispose pas de solutions miracles, de régimes ou de statuts dont il suffirait de dégager les règles et de les appliquer mécaniquement aux questions écologiques. Un intense effort d'imagination juridique est nécessaire qui, bien entendu, cherchera à prendre appui sur les données que recèle la tradition. Ce travail est aujourd'hui entamé ; de divers côtés s'ouvrent des chantiers juridiques. Du côté du droit public, on exhume le concept de «domanialité » dont on montre les virtualités environnementales ; du côté du droit civil, on redécouvre les « choses communes » de l'article 714 du Code civil et on s'avise des potentialités écologiques que recèlent les mécanismes de l'usufruit, de l'usage et des servitudes. Du côté du droit comparé, on vante les mérites du trust anglo-saxon, qui permet notamment d'instaurer un gardien (trustee) de la nature, chargé de sa protection et de sa gestion avisée au bénéfice du public présent et à venir. Ailleurs encore, on évoque la constitutionnalisation d'un "droit fondamental à l'environnement », tandis que d'autres plaident pour l'intégration du souci écologique dans les composantes de l'intérêt général, ce qui, dans la balance des intérêts que pratique journellement le juge, servirait de contrepoids bienvenu aux pressions qu'exercent les intérêts économiques et sociaux traditionnels (Ost, 2003, p. 307). 
La notion de patrimoine est le médiateur de toutes ces interrogations légitimes. Elle est susceptible d'entraîner des solutions traditionnelles dans des voies inédites, parce qu'elle conduit du local au global, du simple au complexe, des intérêts individuels aux intérêts collectifs, d'une appropriation statique et univoque de l'espace à la reconnaissance de la multiplicité des usages. C'est ce qui conduit l'auteur à envisager le concept de «transpropriation » (p. 325). Il s'agit de constater que des intérêts profitant à la communauté viennent interférer avec le droit de propriété individuel. Il y a donc une forme de transversalité susceptible de reformuler les termes de la propriété.

Dans la littérature juridique anglo-saxonne, on considère de façon assez générale la notion de garde ou d'intendance (le stewardship) de la nature comme étant la réponse à la question des rapports entre propriété et environnement. Parce qu'on pense cette garde en termes de maîtrise foncière, elle s'avère particulièrement bien correspondre à l'objectif de développement durable. John Alder et David Wilkinson écrivent :

La notion de surintendance [stewardship] [...], qui impose un devoir de garde et peut-être aussi d'amélioration de l'environnement, est étroitement liée à celle de développement durable. Les deux impliquent un souci des générations futures, et les deux requièrent du propriétaire de choisir la manière de promouvoir le développement la plus respectueuse de l'environnement (Alder et Wilkinson, 1999 ; traduit par Mathieu Galey, 2007, p. 13).

Beaucoup considèrent donc que le droit de propriété* en droit civil, dans lequel le propriétaire exerce, (en théorie, mais de moins en moins en pratique, puisqu'on multiplie désormais les restrictions de droit public à l'exercice de la propriété privée), un droit discrétionnaire sur le milieu, n'offre pas l'intérêt de la notion anglo-saxonne de stewardship, laquelle soulignerait, au contraire, les obligations sociales qui s'ajoutent aux droits individuels (Galey, 2007, p. 13).

\section{La théorie structuraliste des maîtrises foncières et fruitières}

Sur la base de travaux de théoriciens économistes (Elinor Ostrom par exemple), les chercheurs en anthropologie juridique (Étienne Le Roy ; Olivier et Catherine Barrière) ont mis au point des tableaux matriciels particulièrement raffinés fondés sur une excellente connaissance des réalités locales des pays en développement mais aussi des sociétés traditionnelles en Europe. L'idée commune est que les milieux et les communautés observées produisent de façon endogénétique de la juridicité, alors que le droit vient de l'extérieur, c'est-à-dire est imposé d'en haut aux communautés locales. Cette théorie joue un rôle appréciable dans la réflexion actuelle.

Les tableaux matriciels s'avèrent d'excellents outils d'analyse des milieuxcommunautés, pouvant aller jusqu'à des niveaux de combinaisons extrêmes ${ }^{15}$. Sans vouloir abuser du paradoxe, on ne peut s'empêcher de relever l'analogie de ces matrices avec ce qu'une administration fiscale doit faire pour aboutir, dans un cadastre, à définir des classes d'utilisation du sol dans le but de déterminer des tarifications d'impôt foncier. Mais en plus complexe, car les anthropologues prennent également en compte la diversité des acteurs (local ou étranger, homme ou femme, chef ou membre de lignage) et adaptent la classification à la typologie de la société étudiée. Ces auteurs disent se

15. Par exemple, 44 espaces fonciers $\times 5$ niveaux d'acteurs $=220$ combinaisons potentielles chez Olivier et Catherine Barrière, 2002, p. 298-299. 
situer dans le sillage de la notion de stewardship ou gouvernance du foncier*. Ils sont d'ailleurs habités par des représentations négatives fortes de la Modernité rejetant le formalisme et estimant que «Le passage à la propriété foncière n'est en rien inéluctable... » (Barrière, 2002, p. 279). Concernant le système de droit, les anthropologues du droit agissent comme si le droit latin imposait un contenu et dictait la marche à suivre pour sélectionner arbitrairement un ayant droit parmi plusieurs, d'où le rejet ; tandis qu'ils agissent comme si le droit anglo-saxon proposait une forme libre (les faisceaux de droits) dans laquelle on pourrait mettre tout ce qu'on veut.

Néanmoins ces chercheurs proposent des normes! En ce domaine, l'originalité de l'anthropologie juridique à la française est de compenser son affranchissement des niveaux normatifs externes habituels (le droit, les institutions) par une formalisation interne très poussée. Au lieu du droit, institutionnalisant de l'extérieur, la juridicité, auto-organisationnelle, mais en réinterprétant les théories économiques ostromiennes à l'aune de la rationalité française ou latine par la dimension structuraliste (décrire la hiérarchie des acteurs et des milieux et pas seulement à leur mise en réseau) et par une lecture possibiliste, classique en histoire, en sociologie et en anthropologie, en considérant que l'espace est un gisement de contraintes que les sociétés mobilisent différemment selon ce qu'elles veulent mettre en avant.

\section{Approche mésologique de la notion de propriété}

La thèse de la juriste Sarah Vanuxem constitue aujourd'hui, une des avancées les plus significatives qui soient. Elle consiste à poser le principe d'une inversion complète en considérant que les choses ne sont pas des biens mais des milieux ce qui change la conception du propriétaire. Selon elle, on a aujourd'hui la possibilité d'aller vers une conception mésologique des choses, c'est-à-dire de concevoir des choses, constituant des milieux (d'où la qualification de mésologique) qui soient « saisis par la propriété » au sens de saisis par les droits qu'on nomme propriété.

Elle prend l'exemple de l'usufruit. Dans l'article 578 du Code civil qui donne à l'usufruitier le droit de jouir des choses comme le propriétaire lui-même mais «à la charge d'en conserver la substance », ce qu'il faut conserver c'est non seulement la substance corporelle des choses, mais aussi leur destination, c'est-à-dire l'usage auquel elles sont affectées : par exemple si le propriétaire loue un local destiné à l'habitation, le preneur n'a pas le droit d'en faire un local professionnel. Mais la destination est-elle seulement celle «voulue » par le propriétaire (la volonté de l'homme de l'article 579) ? Le législateur voit plus large et dans les articles qui suivent l'article 578, il parle aussi de l'usage des lieux, ou de l'usage constant des propriétaires (art. 590, 591). L'usufruitier peut aussi demander à la justice de l'autoriser à affecter un nouvel usage. Autrement dit, d'autres destinations peuvent se déduire de situations extérieures à la seule volonté du propriétaire. On doit donc revenir à la signification originelle et juridique du mot chose : « ce avec quoi on a affaire dans l'usage ». D'où la métaphore de Sarah Vanuxem : les choses appropriées constituent alors une « demeure » pour ceux qu'elles rassemblent et qui séjournent en elles. Il s'agit donc de concevoir les choses comme des milieux, c'est-à-dire les lieux qui rassemblent.

On quitte la notion de propriétaire-souverain pour celle de propriétaire-habitant (le propriétaire n'étant qu'un habitant privilégié) et on donne toute leur place aux limites envisagées comme réserve dans le texte de l'article 544. Et on peut inverser la proposition 
habituelle : ce n'est pas la propriété qui sort des entrailles de la communauté (la formule est du civiliste* Frédéric Zénati-Castaing), mais c'est la communauté qui séjourne au sein des choses et n'en sort jamais. Ainsi, les choses ne quitteraient jamais une espèce d'usage commun. La propriété ne les absorberait jamais, au mieux elle peut exercer une saisine*. La théorie classique qui refuse que l'appropriation porte sur des droits se voit écartée. Ainsi, quiconque a un droit d'usage ou d'usufruit sur la chose d'autrui a déjà une place dans ou sur la chose d'autrui, tandis que le propriétaire d'un droit sur sa propre chose occupe une place dans la chose qui ne se trouve pas contenue dans celle d'un autre.

Il faut alors concevoir la communauté des choses comme un état actuel et non pas un état primitif. Cela revient à rompre avec l'ancienne théorie des stades qui a longtemps formaté la pensée historique, juridique et sociologique, et dans laquelle la communauté était au début, avant l'appropriation. De même, concevoir des choses-milieux, c'est faire que les choses fassent carte ou rhizome avec d'autres choses expérimentées.

\section{L'effet des nouvelles pratiques foncières sur le droit}

Cette partie recense quelques choix significatifs de nouvelles pratiques foncières, qui ne construisent pas, pour autant, une approche commune en Europe. Ce sont des évolutions nouvelles et même contradictoires par rapport au tableau qui a été dessiné jusqu'ici dans cet article.

\section{Réduire la spécificité ou l'insularité de tel ou tel droit}

Les deux premiers exemples ont pour but de mettre en évidence une tendance à la réduction de la situation d'insularité des familles de droit et leur interpénétration progressive. Car si utiliser la notion de familles de droit (anglo-saxon, latin, germanique, etc.) est utile pour écrire l'histoire, cette base s'affaiblit chaque jour un peu plus en présence de la mondialisation* du droit (Halpérin, 2009, notamment p. 339 sq).

En Angleterre, et jusqu'à une date très récente, le cadastre (juridique autant que fiscal) a été constamment rejeté, notamment par l'aristocratie, par crainte de l'intervention des pouvoirs publics dans le domaine privé. Cette tendance s'inverse aujourd'hui. Une première évolution a été l'introduction d'un système d'enregistrement des titres fonciers par la loi de 1925. Elle est aujourd'hui complétée par différentes lois de 1988 et 2002 qui instaurent un registre des biens-fonds, un registre des propriétaires fonciers et un registre des charges, tous trois publics depuis 1988. Cela revient à aller vers un véritable cadastre juridique. Cet enregistrement est obligatoire depuis 1990 et marque la volonté du gouvernement d'aller vers un enregistrement total des terres.

Ce cadastre est complété par un volet cartographique. En effet, depuis 1992, le Land Registry coopère avec l'Ordnance Survey et avec le Valuation Office pour établir un service pilote d'information foncière dit National Land Information Service (NLIS). La réforme introduit trois principes qui, s’ils étaient strictement appliqués (ce qui n'est pas le cas), constitueraient un changement radical de perspective :

1. Le registre reflète tous les faits relatifs au titre foncier (la consultation du registre devant suffire pour connaître l'existence de tiers pouvant détenir des droits de propriété grevant le terrain ; c’est ce qu'on appelle en droit foncier le « principe du miroir ») ; 
2. Mais les trusts sont maintenus à l'écart du registre ;

3. Toute personne qui a subi un dommage à la suite d'une erreur du registre doit être indemnisée (c'est le « principe de l'assurance »).

Cependant diverses limitations existent : le principe n'est pas d'enregistrer de manière systématique et préalable les biens-fonds, mais de profiter d'une transmission pour réaliser l'enregistrement foncier et fiscal du bien, de manière publique, ce qui fait qu'aujourd'hui seule une moitié des biens-fonds est enregistrée en Angleterre et au Pays de Galles. Malgré ces limites, on peut qualifier de relativement profond le changement que cette adoption d'un cadastre juridique représente, compte tenu de la résistance historique de la population, et principalement de ses élites, à l'enregistrement piloté par l'État.

Voici un autre exemple, l'introduction de la fiducie* dans le droit latin (Québec, Luxembourg, Italie, France), évoqué à partir du cas français. La fiducie française ou " propriété affectée » est un mécanisme de gestion des patrimoines, un équivalent du trust. C'est un mécanisme d'abord réservé aux personnes morales payant l'impôt sur les sociétés (loi de 2007) puis étendu aux personnes physiques en 2008. Il associe, au moins dans le principe, un constituant, un fiduciaire et un bénéficiaire. Il sert à transférer des biens afin de constituer des sûretés ou de permettre la gestion pour le compte d'un bénéficiaire vulnérable. Mais alors que le trust anglais est un acte unilatéral décidé par le constituant et créant deux propriétés simultanées (celle du settlor ou constituant; celle du trustee ou fiduciaire) au service d'un troisième, le bénéficiaire, au contraire en France, c'est un contrat de transfert de propriété, limité dans le temps et limité dans l'usage que le fiduciaire peut faire du bien transmis. Il ne touche pas à l'unité de la propriété (ou monisme de la propriété définie par le Code civil), car seul le fiduciaire a la propriété. Mais, en revanche, il met à mal la notion, cardinale en droit français, d'indivisibilité du patrimoine puisque le fiduciaire gère le patrimoine dont il reçoit la propriété en le séparant expressément de son propre patrimoine, afin de rendre des comptes au constituant.

C'est parce qu'elle dresse un mur de protection autour des biens (en les ayant transférés au fiduciaire), que l'institution a provoqué des résistances dans les pays à forte tradition fiscale comme la France. En protégeant l'actif contre les créanciers, elle peut aussi donner l'impression de vouloir échapper à l'impôt.

\section{Des outils qui actent le changement du droit}

Dans les pays occidentaux, la propriété change sous la pression de la domanialité publique. Elle est de plus en plus grevée de contraintes de droit public national et surtout de droit public supranational (le droit européen ; les préconisations mondiales). Ces contraintes la font sensiblement évoluer vers autre chose que ce qu'on a connu et formalisé lors de la modernité. Car ce qui est nouveau, c'est l'accumulation des contraintes de droit public sur la propriété privée, dans le respect formel de celle-ci. Autrement dit, la propriété renoue avec son pluriel (ou l'un de ses pluriels) et redevient, de fait, simultanée.

Des outils juridiques apparaissent pour nommer et mesurer ces contraintes, la Suisse venant d'en donner un exemple. Ce pays a créé, en 2009, le cadastre des restrictions de droit public à la propriété foncière (RDPPF) $)^{16}$, que cinq cantons ont ouvert en 2014 ,

16. Les premiers cantons concernés sont : Berne, Neuchâtel, Nidwald, Thurgovie et Zurich. Voir le site de présentation de ce cadastre sur le site cadastre.ch, URL : < https://www.cadastre.ch/fr/manual-oereb. html>. 
l'ensemble du territoire de la Suisse devant être couvert en 2019. Il s'agit d'un système d'information géographique (ou foncier) juridique qui peut dire au citoyen intéressé par l'acquisition d'un immeuble, toutes les restrictions de droit public qu'il va rencontrer, parce que les différentes administrations sont tenues de renseigner la base informatique. Cela revient à inverser le principe selon lequel nul n'est censé ignorer la loi. Ici, le principe devient : devant l'accumulation des restrictions dont est grevée la propriété privée, c'est à l'État à faire connaître aux citoyens toutes les réglementations et les limites à l'exercice du droit de propriété*. Au-delà de la nouveauté de la technique de "forage » (métaphore pour nommer la méthode d'interrogation de la base SIG ou SIF), ce qui est nouveau, c'est le fait que ces restrictions puissent devenir en quelque sorte le centre et non la marge, qu'on puisse définir le droit autant par rapport à elles que par rapport au droit de propriété privée. En quelque sorte, le spatial, véhicule de l'intérêt collectif, l'emporte ici sur l'ancienne règle de droit écrit ou au moins l'équilibre fortement. De ce fait on mesure le pas fait en direction d'une conception plurielle de la propriété, car c'est, dans le fond, une forme de progrès de la domanialité publique et une restriction de la propriété privée.

Cet exemple fait toucher du doigt le progrès d'une nouvelle conception de l'outil cadastral qui est susceptible d'aider à la prise de conscience de la transformation du droit. C'est ce qu'on nomme le cadastre à multiples usages (Mehrzweck-Kataster en allemand ; multipurpose cadastre en anglais), cadastre qui ne sert pas exclusivement à établir la fiscalité ou à prouver la propriété, mais qui sert aussi à d'autres fonctions sociales : planification territoriale ; gestion de l'environnement ; aménagement agricole ; connaissance des restrictions de droit public ; etc. Cet élargissement de la définition du cadastre conduit logiquement à dissocier l'établissement de la base cartographique des usages qu'on en fait, car la base cartographique, quelquefois dénommée " plan foncier de base », est utilisée dans diverses fonctions.

Enfin, le portage foncier bouleverse les rapports sociaux créés autour de la terre. On désigne par cette expression la situation dans laquelle on dissocie la propriété du foncier de l'exploitation agricole. Le but est d'alléger la charge économique de l'exploitant en le libérant du poids de l'investissement foncier ou en l'atténuant. On fait donc porter la terre par d'autres que par l'exploitant. Le portage peut être familial ou sociétaire. Dans ce dernier cas, à côté des situations de portage pour réguler le foncier (ex. : les sociétés d'aménagement foncier et d'établissement rural ou SAFER), on voit apparaittre des sociétés de portage à but spéculatif et des associations pour le portage foncier non spéculatif, comme Terre de Liens. La généralisation du portage est une tendance émergente, en raison de la chute du nombre des agriculteurs, qui pourrait conduire, à terme, à la création de puissantes entreprises concentrant la ressource foncière pour la mettre à disposition.

\section{Conclusions}

L'ampleur de la réécriture en cours du récit* du droit de la propriété comme la multiplicité des expériences qui, par la pratique, en changent actuellement les contours tendent, paradoxalement, à relativiser les débats autour de la notion même. Car chacun constate qu'on passe actuellement d'une époque où les débats se terminaient le plus souvent par des choix politiques (acceptés ou contestés, selon les cas), à une nouvelle 
époque où l'horizon est l'état de controverse sans fin, la controverse se perdant dans le faisceau de ses propres contradictions. Par exemple, si on pense qu'il faut réouvrir la propriété et donner du poids à chacune des utilités qu'elle rassemble, comment conciliera-t-on cette idée avec le risque d'une conception utilitaire du droit par celui qui exploite et ne possède pas ? Car l'usufruitier, qui profite d'une utilité sans avoir de préoccupation d'ensemble, peut n'être tenté que par l'intérêt immédiat et non pas par le long terme, puisqu'il n'est pas le propriétaire. Faut-il alors, comme Hubert Bosse-Platière l'évoque dans son propre chapitre, suivre Jean Carbonnier (2001, p. 384) et affirmer, à sa suite, que seule la propriété familiale suppose la continuité des soins sur plusieurs générations, parce qu'elle est rassemblée dans les mêmes mains ? Mais alors, comment défendre le modèle familial si, dans le même temps, ce sont les mêmes qui défendent aussi l'éclatement de la propriété, par exemple par séduction envers la notion de faisceaux de droit? Voilà typiquement deux controverses (celle sur l'unité ou l'éclatement de la propriété, et celle sur l'intérêt ou non du modèle familial) qui se croisent et, finalement, bloquent la recherche d'une solution.

Cependant, ces débats occupent, comme souvent, beaucoup d'importance et, de ce fait, contribuent aussi à transformer la réalité. Ainsi, d'un côté on veut éprouver jusqu'à l'extrême les conséquences de l'aplatissement du monde et dérouler une conception uniforme et prétendument apolitique des droits de propriété en demandant aux autres droits et conceptions de s'adapter progressivement à cette norme qui ne veut surtout pas en être une : c'est, par exemple, le paradigme de la Banque mondiale. De l'autre, on s'engage dans une réflexion sur les communs, sans prendre la peine d'en poser solidement les bases historiques, et en l'exploitant pour en faire un levier de changement politique vers un monde où... on réduirait la part du politique par la subsidiarité*, c'est-à-dire où on ferait de la politique sans droit ni institutions, par la seule vertu autorégulatrice de la « gouvernance ».

L'une des issues possibles de cette tenaille idéologique est d'engager une description et une analyse des systèmes fonciers formalisés afin d'en connaître le plus consciemment possible les fondements et, sans en espérer plus qu'ils ne peuvent donner, d'en éprouver la capacité propositionnelle. Car la question actuelle la plus difficile est la recherche d'instances de régulation du régime de contrat généralisé qui est celui dans lequel l'économie et le droit nous placent chaque jour un peu plus. En effet, le modèle du contrat se donne aujourd'hui comme horizon des relations et l'illusion de son efficience est d'autant plus forte qu'il est difficile de faire mieux et plus démocratique que l'expression de la volonté des parties. Sauf qu'en matière foncière, cette liberté n'a jamais paru suffisante seule et on l'a longtemps encadrée. Ces protections, nationales ou européenne, cèdent progressivement.

Si ce n'est plus l'État qui régule, on est alors tenté de chercher dans les formes issues de sa fragmentation (établissements publics, agences, etc.) les institutions qui pourront garantir ce contrôle, tout en devant obéir aux directives d'autres institutions, supranationales cette fois, telles que l'OMC, la Banque mondiale, le FMI, l'OCDE, dont la fonction est de définir les critères d'évaluation économique de cette contractualisation, jusqu'aux agences d'évaluation, qui s'attribuent la fonction d'en apprécier le respect.

Ce passage du statut au contrat, d'un droit de l'institutionnalisation à un droit de la contractualisation, n'est pas une question mineure, car, dans un monde dominé par l'accroissement vertigineux des inégalités, il faut être attentif à tous les signes qui indiquent que le contrat réintroduit ou risque de réintroduire de nouvelles formes de dépendance. 


\section{Bibliographie}

Adler John et Wilkinson David, 1999, Environmental Law and Ethics, Londres, Mac Millan.

Assier-Andrieu Louis, 1996, Le droit dans les sociétés humaines, Paris, Nathan.

Aubin David, Nahrath Stéphane et Varone Frédéric, 2004, Paysage et propriété : patrimonialisation, communautarisation ou pluridomanialisation, Chavannes-Lausanne, IDHEAP.

BARriÈRe Olivier et Catherine, 2002, Un droit à inventer. Foncier et environnement dans le delta intérieur du Niger (Mali), Paris, IRD Éditions.

Berman Harold Joseph, 2011, Droit et Révolution : l'impact des réformes protestantes sur la tradition juridique occidentale, Paris, Fayard.

Berque Augustin, 2000, Écoumène. Introduction à l'étude des milieux humains, Paris, Belin.

BROCHU François, 1997, La publicité foncière et la prescription acquisitive en droit civil québécois à la lumière du droit français, suisse, allemand et australien, thèse de doctorat, Aix-en-Provence/Marseille, Université Aix-Marseille 3.

Caillé Alain, 1989, Critique de la raison utilitaire, Paris, La Découverte.

Carbasse Jean-Marie, 2002, Manuel d'introduction historique au droit, Paris, PUF.

Carbonnier Jean, 2001, Flexible droit. Pour une sociologie du droit sans rigueur, Paris, LGDJ.

Centre d'Études sur la CoOpération juridique internationale, 2012, Les modèles propriétaires au $X X I^{e}$ siècle, actes du colloque international en l'honneur de Henri-Jacques Lucas (Poitiers, 10 et 11 décembre 2009), Poitiers/Paris, Presses universitaires de Poitiers/LGDJ.

Centre français de droit comparé, 1997 (juillet-septembre), « Actualités de la propriété privée dans les pays d'Europe centrale et orientale et en Chine, colloque de 1996 », Revue internationale de droit comparé, vol. 49, n 3, p. 527-625.

Chevalet Catherine (éd.), 1991, Un droit inviolable et sacré, la propriété, actes du colloque de l'ADEF (Paris, 13 et 14 novembre 1989), Paris, ADEF.

Chouquer Gérard, 2012, Terres porteuses. Entre faim de terres et appétit d'espace, Paris/Arles, Errance/Actes Sud.

Colin Jean-Philippe, 2013, Marchés fonciers et concentration foncière. La configuration de «tenure inversée » (reverse tenancy), Montpellier, Agropolis.

— 2014 (juillet-décembre), « La concentration foncière par la tenure inversée », Études rurales, $\mathrm{n}^{\circ} 194$, p. 203-218.

Comby Joseph, 1991, «L'impossible propriété absolue », in Chevalet Catherine (éd.), Un droit inviolable et sacré, La propriété, actes du colloque de l'ADEF (Paris, 13 et 14 novembre 1989), Paris, ADEF, p. 9-20.

Comité technique « Foncier et Développement »(AFD-MAEE), 2010 (juin), Les appropriations de terres à grande échelle. Analyse du phénomène et propositions d'orientations, Paris, Ministère des Affaires étrangères. 
CORIAT Benjamin (dir.), 2015, Le retour des communs. La crise de l'idéologie propriétaire, Paris, Les Liens qui libèrent.

Dardot Pierre et Laval Christian, 2014, Commun. Essai sur la révolution au XXI siècle, Paris, La Découverte.

— 2011, La nouvelle raison du monde. Essai sur la société néolibérale, Paris, La Découverte.

Demélas Marie-Danielle et VIVIER Nadine (dir.), 2003, Les propriétés collectives face aux attaques libérales (1750-1914), Rennes, Presses universitaires de Rennes.

Fenet Pierre-Antoine et Ewald François (éds), 1989, Naissance du Code civil. Travaux préparatoires du Code civil, Paris, Flammarion.

Friedman Thomas, 2006, La terre est plate. Une brève histoire du XXIe siècle, Paris, Perrin.

Galey Matthieu, 2007, «La typologie des systèmes de propriété de C. R. Noyes. Un outil d'évaluation contextualisée des régimes de propriété privée, publique et commune », in Eberhard Christoph (dir.), Enjeux fonciers et environnementaux. Dialogues afro-indiens, Pondichéry, Institut français de Pondichéry, 2007, p. 89-125.

GARnER Guillaume, 2005, État, économie, territoire en Allemagne. L'espace dans le caméralisme et l'économie politique, 1740-1820, Paris, Éditions de l'EHESS.

Gau-Cabée Caroline, 2006, Droits d'usage et Code civil. L'invention d'un bybride juridique, Paris, LGDJ.

Grey Thomas C., 1980, "The Desintegration of Property », in Pennock Roland et Chapman John (éd.), Nomos XXII, Property, New York, New York University Press, p. 69-85.

Grossi Paolo, 1977, Un altro modo di possidere. L'emersione di forme alternative di proprietà alla coscienza giuridicha post-unitaria, Milan, Giuffrè.

Halperin Jean-Louis, 2009, Profils des mondialisations du droit, Paris, Dalloz.

— 2008, Histoire du droit des biens, Paris, Economica.

Hardin Garrett, 1968, «La tragédie des communaux » [traduit en français par Michel Roudot], Science, $\mathrm{n}^{\circ} 162$ [en ligne], URL : <http://lanredec.free.fr/polis/ art_tragedy_of_the_commons_tr.html $>$.

Harribey Jean-Marie, 2011 (janvier), « Le bien commun est une construction sociale. Apports et limites d'Elinor Ostrom », L'économie politique, $\mathrm{n}^{\circ} 49$ [en ligne], URL: $<$ http ://www.alternatives-economiques.fr/le-bien-commun-est-une-construction_fr_art_1072_53003.html>.

Kain Roger J. P., Baigent Elizabeth, Fletcher David, 2008, « Relevé cadastral en Angleterre et au Pays de Galles : la propriété privée, l'État et les plans manquants », in Bourillon Florence, Clergeot Pierre et Vivier Nadine (dir.), De l'estime au cadastre en Europe, Les systèmes cadastraux aux XIX et XX siècles, Paris, Comité pour l'histoire économique et financière de la France, p. 21-46.

KILZI Jean, 2002, Le cadastre, le registre foncier et les propriétés foncières au Liban, Beyrouth, Chemaly \& Chemaly Press. 
LE Roy Étienne, 2011, La terre de l'autre. Une anthropologie des régimes d'appropriation foncière, Paris, LGDJ.

Maganzani Lauretta, 2007 [2002], Formazione e vicende di un'opera illustre. Il corpus iuris nella cultura del giurista europeo, Turin, G. Giappichelli.

Marne Henri Sumner, 1871, Village communities in the East and West, Londres, s. n. [trad. française dans Études sur l'histoire du droit, Paris, Ernest Thorin, 1889].

Mari Éric (de) et Taurisson-Mouret Dominique (éd.), 2016 (novembre), L’Empire de la propriété. L'Impact environnemental de la norme en milieu contraint (III), Paris, Victoires Éditions.

MARXer Birgit, 2003, Idéologie foncière en Russie du XVIe siècle à aujourd'bui, Paris, L'Harmattan, Paris.

Maurel Marie-Claude, 2012 (juillet-décembre), « La grande maille agraire en Europe centrale : un invariant spatiotemporel ?", Études rurales, n 190 , p. 25-48.

- 1994, La transition postcollectiviste. Mutations agraires en Europe centrale, Paris, L'Harmattan.

Mouvement Jeune Notariat, 2012, Le livre et la plume. Publicité foncière et Notariat : quel avenir?, actes du 43 e congrès (Grenade, 17 au 21 octobre 2012), Paris, Mouvement Jeune Notariat.

Noyes Charles Reinold, 1936, The institution of property. A Study of the development, substance ans arrangement of the system of property in modern anglo-amercican law, New York/ Toronto, Longmans/Green and Co.

Ost François, 2003 [1995], La nature hors la loi. L'écologie à l'épreuve du droit, Paris, La Découverte.

Ostrom Elinor, 2010, Gouvernance des biens communs. Pour une nouvelle approche des ressources naturelles, Bruxelles, De Boeck.

Papandréou-Deterville Marie-France, 2004, Le droit anglais des biens, Paris, LGDJ.

Parange Béatrice et Saint-Victor Jacques (de) [dir.], 2014, Repenser les biens communs, Paris, CNRS Éditions.

Patault Anne-Marie, 1989, Introduction historique au droit des biens, Paris, PUF.

RochFeLD Judith, 2015, «Quels modèles juridiques pour accueillir les communs en droit français? ", in CORIAT Benjamin (dir.), Le retour des communs. La crise de l'idéologie propriétaire, Paris, Les Liens qui libèrent, p. 87-105.

Schiavone Aldo, 2008, Ius, L'invention du droit en Occident [traduit par Geneviève et Jean Bouffartigue], Paris, Belin.

Soто Hernando (de), 2005, Le mystère du capital, Paris, Flammarion.

STEIN Wolfgang Hans, 2008, "Les trois âges du cadastre allemand », in BouriLlon Florence, Clergeot Pierre et Vivier Nadine (dir.), De l'estime au cadastre en Europe. Les systèmes cadastraux aux $X I X^{e}$ et $X X^{e}$ siècles, Paris, Comité pour l'histoire économique de la France, p. 73-98. 
— 2007, "L'État de finance et les "familles" de cadastre en Allemagne à l'époque moderne ", in TOuzery Mireille (dir.), De l'estime au cadastre en Europe. L'époque moderne, Paris, Comité pour l'histoire économique de la France, ministère des Finances, p. 81-116.

STEIN Wolfgang Hans et SupIOT Alain, 2005, Homo juridicus. Essai sur la fonction anthropologique du droit, Paris, Seuil.

VANUXEM Sarah, 2012, Les choses saisies par la propriété, Paris, IRJS Éditions.

Vivier Nadine, 2008, «Les débats sur la finalité du cadastre, 1814-1870 », in BouriLlon Florence, Clergeot Pierre et Vivier Nadine (dir.), De l'estime au cadastre en Europe, Les systèmes cadastraux aux XIX et XX siècles, Paris, Comité pour l'histoire économique et financière de la France, p. 191-215.

- 1998, Propriété collective et identité communale. Les biens communaux en France, 1750-1914, Paris, Publications de la Sorbonne.

Williamson Ian, Enemark Stig, Wallace Jude et Rajabifard Abbas, 2010, Land administration for sustainable Development, Redlands (CA), ESRI Press Academic.

XIFAras Mikhail, 2004, La propriété. Étude de philosophie du droit, Paris, Presses universitaires de France.

Yovanovitch Yan, 1895, Des systèmes hypothécaires basés sur les livres fonciers, Grenoble, Impr. Allier père et fils.

ZénAti-CASTAing Frédéric, 2012, « Le crépuscule de la propriété moderne. Essai de synthèse des modèles propriétaires », in CENTRE D'ÉTUDES SUR LA COOPÉRATION JURIDIQUE INTERNATIONALE, Les modèles propriétaires an XXI siècle, actes du colloque international en l'honneur de Henri-Jacques Lucas (Poitiers, 10 et 11 décembre 2009), Poitiers/Paris, Presses universitaires de Poitiers/LGDJ, p. 225-238.

Zénati-Castaing Frédéric et Revet Thierry, 2008 [1988], Les biens, Paris, Presses universitaires de France. 


\section{Table des matières}

\section{Introduction}

Gérard Chouquer et Marie-Claude Maurel 9

Première partie : Évolutions et révolutions dans les politiques agricoles France

1 Les révolutions agricoles contemporaines en France Sophie Devienne.

2 L'espace politique agricole français (1945-2015)

Clémence Guimont et Bruno Villalba.....

3 Le droit du sol et le sang de la terre Hubert Bosse-Platière

\section{Europe}

4 La politique agricole commune : un compromis européen en crise Aurélie Trouvé et Gilles Bazin.

5 Réappropriation foncière dans les campagnes d'Europe centrale Marie-Claude MAUREL

6 Ressource foncière, rente et mutations des agricultures méditerranéennes Pascal Chevalier et Guillaume LACQuement. 


\section{Seconde partie : Interrogations autour des modèles}

7 Des entreprises agricoles « aux allures de firmes » François Purseigle, Guilhem AnZalone, Geneviève NGuYen et Bertrand Hervieu 163

8 Le modèle de l'agriculture familiale en France Hubert COCHET

9 L'inquiétant modèle du « travail saisonnier » en Europe Gérard Chouquer

10 Interrogations théoriques autour de la propriété et du foncier en Europe Gérard Chouquer

Liste des sigles et acronymes 257

Lexique 261

Index géographique 277

Ont collaboré à l'ouvrage 281

Table des figures.. 289 
Imprimerie Messages

111, rue Nicolas Vauquelin - 31100 Toulouse

Dépôt légal : premier trimestre 2019

Imprimé en France 
L'ouvrage propose un tour d'horizon des évolutions les plus récentes du foncier et de l'agriculture en Europe. Une première partie caractérise les évolutions et les révolutions des politiques agricoles, étudiées du point de vue du droit, de la science politique, de l'agronomie et de la géographie, en mettant l'accent sur la fin d'une phase protectionniste, et avec deux éclairages régionaux, en Méditerranée et dans l'Europe médiane. Une seconde partie interroge les modèles agricoles et fonciers : I'agriculture familiale, l'agriculture de firme, le travail saisonnier, les mutations de la propriété et l'évolution vers le portage foncier.

Gérard CHOUQUER, historien, est directeur de recherches au CNRS honoraire et membre correspondant de l'Académie d'agriculture de France.

Marie-Claude MAUREL, géographe, est directrice d'études à l'EHESS et membre de l'Académie d'agriculture de France.

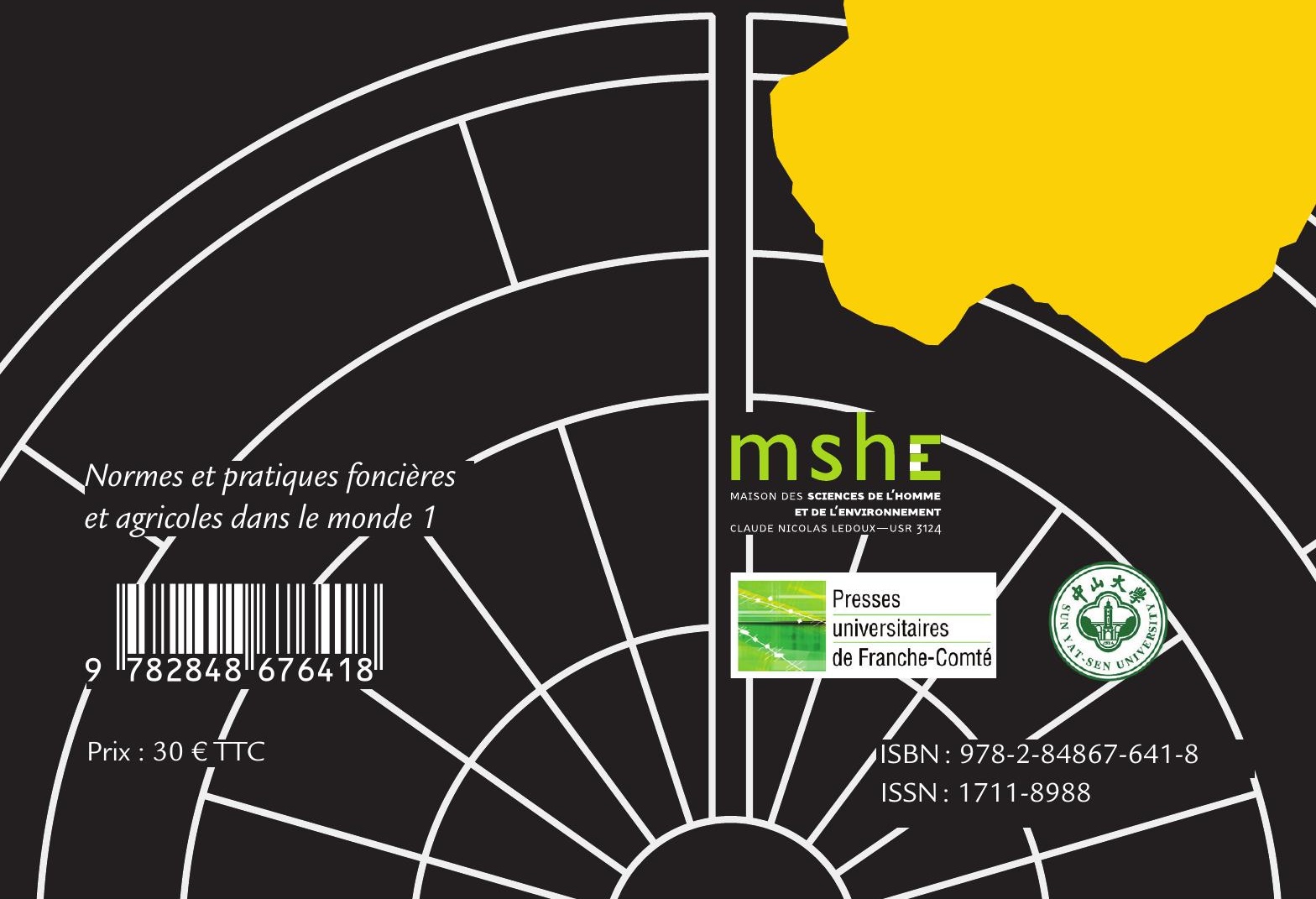

